\title{
SISTEMAS LINEARES
}

Uma solução na norma Chebyshev

\section{Edméa Cássia Baptista}

Orientador: Prof. Dr. Marcos Nereu Arenales

Dissertação apresentada ao Instituto de Ciências Matemáticas

de São Carlos, da Universidade de São Paulo, para obtenção do Título de Mestre em Ciências da Computação e Matemática Computacional.

\section{São Carlos}

Dez/93 


\section{RESUMO}

Neste trabalho, estudamos o problema de determinar uma solução aproximada de um Sistema Iinear Inconsistente, segundo a norma de Chebyshev.

A partir de uma motivação em Engenharia Mecânica, denominada Planicidade de superfícies, modelamos o problema, o qual é resolvido usando uma especialização do método simplex. Propomos uma estrutura de dados, onde as colunas da matriz do "Modelo Linearizado" são geradas, ao invés de serem armazenadas, e apresentamos alguns estudos computacionais.

Finalizando, fazemos a revisão do artigo "strict Chebyshev Approximation for General Systems of Linear Equations" de J. P. Thiran e S. Thiry, 1987 . 


\section{ABSTRACT}

This work studies a problem which consists in to determine a approximate solution of a Inconsistent Linear System according the Chebyshev norm.

From a problem in Mechanic Engineering called Flatness of Surfaces, we model the problem, which is solved using a specialization Simplex method. Then we propose a information structure, where the columns of the matrix of the "Linearized Model" are generated not stored. After this we show some computational studies.

Finishing, we make a revision on the "Strict Chebyshev Approximation for General Systems of Linear Equations" a paper of J.P. Thiran and S. Thiry, 1987. 


\title{
À DEUS
}

\begin{abstract}
Que nos acalentou no limiar da madrugada; nas nossas horas de angústia, cheia de incertezas e saudades, naqueles momentos em que tudo parecia nublado, ELE sempre esteve presente e sabemos que sua força nos ajudou, fazendo-nos chegar à realização que ostentamos agora.
\end{abstract}

A TI que nos deu a aula da vida.

$$
(\ldots)
$$


Aos meus pais com amor. 


\section{AGRADECIMENTOS}

Ao Prof. Dr. Marcos Nereu Arenales, do ICMSC-USP, que com muita paciência e disponibilidade me orientou na elaboração desta dissertação.

Ao Prof. Dr. Álvaro José Abackerli, da EESC-USP, pela atenção e colaboração no desenvolvimento deste trabalho, e participação na banca do exame de qualificação e defesa.

Aos professores que fizeram parte da banca do exame de qualificação e ao Prof. Dr. Messias Meneguette Junior, da UNESP-PRES. PRUDENTE, pela participação na banca de defesa.

Aos professores e colegas da UNESP-BAURU, pela colaboração direta e indireta; em especial à Andréa, Denise e Vanilda.

Aos professores e funcionários do ICMSC-USP, pela ajuda na confecção deste trabalho.

A minha mãe, pela "vida", incentivo e apoio nos momentos certos.

A Edna, Eli e Erica, pela força.

A todos os "amigos", que conviveram comigo durante esta fase, pelo companheirismo e apoio. 
ÍNDICE

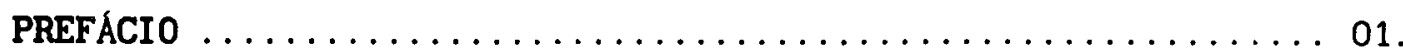

\section{CAPITULO 1 INTRODUÇÃo}

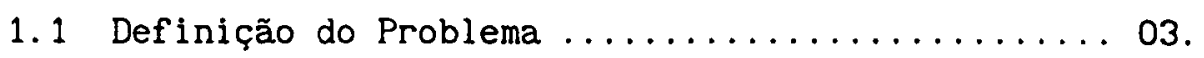

1.2 Uma Aplicação na Metrologia .............. 06.

\section{CAPITULO 2 RESOLUÇÃO VIA PROGRAMAÇÃO LINEAR}

2.1 Problema de Programação Linear Equivalente...... 11.

2.2 O Problema Dual...................... 12.

\section{CAPITULO 3 ESTUDO DE CASO: PLANICIDADE DE SUPERFICIES}

3. 1 Estrutura de Dados................... 14 .

3.2 Solução Básica Inicial................. 17.

3.2.1 Posto de B.................... 20.

3.3 Vetor Multiplicador................. 22.

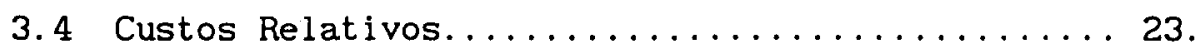

3.4 .1 Observação.................... 25.

3.5 Algoritmo Simplex Aplicado ao Problema de

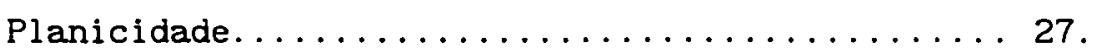

3.6 Experiência Computacional.............. 29.

\section{CAPITULO 4 UM MÉTODO ASCENDENTE}

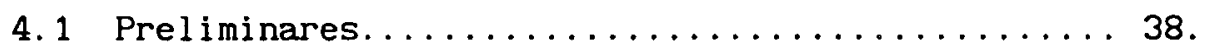


4.1.1 Definição de H-Conjuntos e H-Conjuntos Minimais para Sistemas Lineares......... 38.

4.1.1.1 Propriedades de H-Conjuntos Minimais................ 41.

4.1.2 Caracterização das Soluções Otimas Usando H-Conjuntos Minimais.......... 44.

4.2 Decomposição Canônica da Matriz............ 50.

4.3 Partição do Sistema Relativo a um H-Conjunto Minimal......................... 54.

4.4 Troca Entre H-Conjuntos Minimais............ 59.

4.5 Determinação do H-Conjunto Minimal........... 68.

4.6 Algoritmo e Exemplos Numéricos............ 70.

4.6.1 Algoritmo................... 70.

4.6.2 Exemplos Numéricos.............. 75.

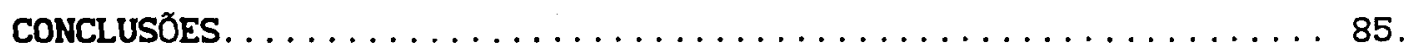

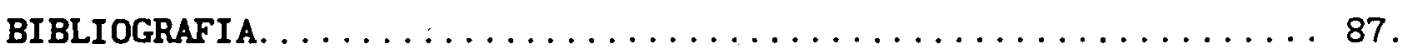




\section{PREFÁCIO}

O objetivo deste trabalho é determinar a solução aproximada de um Sistema Linear Inconsistente, usando a Norma de Chebyshev. Existem vários métodos para solucionar este problema, como o Método de Barrodale e Phillips, o Método de Stiefel, o Método Simplex, entre outros [3].

Neste trabalho fazemos dois estudos, um deles é um estudo de caso, em Engenharia Mecânica, na área de Metrologia, o qual é denominado PLANICIDADE DE SUPERFICIES. O outro é um estudo teórico, onde é apresentado um algoritmo para o problema proposto, o qual explora partições do sistema e decomposições da matriz.

Para o caso de PLANICIDADE DE SUPEFICIES, optamos por usar uma especialização do método Simplex, devido à sua simplicidade de implementação e apresentamos alguns resultados computacionais.

No Capítulo 1, seção 1.1, fazemos uma introdução ao problema de resolução de Sistemas Lineares Inconsistentes; na seção 1.2, apresentamos o problema de PLANICIDADE modelado.

No Capítulo 2, mostramos que o problema aqui estudado pode ser visto como um Problema de Programação Linear e exploramos sua estrutura Dual.

No Capitulo 3, desenvolvemos todos os passos da especialização do método Simplex, aplicado ao problema de Planicidade e fornecemos um algoritmo estruturado, bem como, os resultados computacionais obtidos 
por esse método, comparados com os resultados obtido pelo método dos Mínimos Quadrados.

Finalmente, no Capítulo 4, fazemos a revisão do artigo: "STRICT CHEBYSHEV APPROXIMATION FOR GENERAL SYSTEMS OF LINEAR EQUATIONS", de J.P. Thiran e S.Thiry, 1987, onde demonstramos vários teoremas propostos no artigo. 


\section{CAPÍTULO 1}

\section{INTRODUC̣ÃO}

Neste Capítulo introduzimos o problema a ser estudado neste trabalho e fazemos uma aplicação em Metrologia, como motivação.

\subsection{Definição do Problema.}

Consideremos o sistema de equações lineares:

$$
A x=b,
$$

onde: $A \in \mathbb{R}^{\operatorname{mxn}}, \quad b \in \mathbb{R}^{m}$ e $x \in \mathbb{R}^{n}$.

Suponhamos, entretanto, que o sistema (1.1) seja inconsistente, isto é, não existe $x \in \mathbb{R}^{n}$ tal que a igualdade se verifique.

O problema a ser abordado neste trabalho consiste em determinar uma solução $\mathbf{x} \in \mathbb{R}^{\mathrm{n}}$, a qual minimiza a norma de Chebyshev do vetor residuo, dado por:

$$
r(x)=b-A x .
$$

Ou seja, queremos determinar $\hat{x} \in \mathbb{R}^{n}$ tal que 


$$
\begin{aligned}
& \min \|r(x)\|_{\infty}=\|r(x)\|_{\infty}=\alpha, \\
& x \in \mathbb{R}^{n}
\end{aligned}
$$

onde $\|r(x)\|_{\infty}=\max \left\{\left|r_{i}(x)\right| ; 1 \leq i \leq m\right\}$; o vetor $\hat{x}$ é denominado solução Chebyshev para (1.1). O problema (1.2) pode ser escrito, equivalentemente, como um problema de Programação Linear, como veremos no Capítulo 2.

Outras normas poderiam ser definidas, como a norma Euclidiana, a qual produziria a bem estudada solução "Mínimos Quadrados".

Consideremos, por exemplo, o problema de ajustar uma reta $\mathbf{y}=\alpha \mathbf{x}+\beta$ a um conjunto de pontos:

$$
\begin{array}{c|ccc}
\mathbf{x} & \mathrm{x}_{1} & \cdots & \mathrm{x}_{\mathrm{m}} \\
\hline \mathrm{y} & \mathrm{y}_{1} & \cdots & \mathrm{y}_{\mathrm{m}}
\end{array}
$$

Gostaríamos que os valores $\alpha$ e $B$ fossem tais que:

$$
\left\{\begin{array}{l}
\alpha x_{1}+\beta=y_{1} \\
\alpha x_{m}+\beta=y_{m}
\end{array} .\right.
$$

Porém, os dados podem não definir uma reta e, portanto, o sistema acima é inconsistente. Definindo-se $r_{1}(\alpha, \beta)=y_{1}-\alpha x_{1}-\beta$, com $i=1, \ldots, m$, temos $\circ$ problema de determinar $\alpha$ e $\beta$ tais que:

$$
\min \|r(\alpha, \beta)\|_{\infty}
$$


onde $\left[\begin{array}{l}\alpha \\ \beta\end{array}\right] \in \mathbb{R}^{2}$.

As Fig. 1,2 e 3 ilustram o comportamento para a solução, com diferentes normas escolhidas:

- Norma $L_{1}:\|\Gamma(\alpha, \beta)\|_{1}=\sum_{1=1}^{m}\left|r_{1}(\alpha, \beta)\right|$.

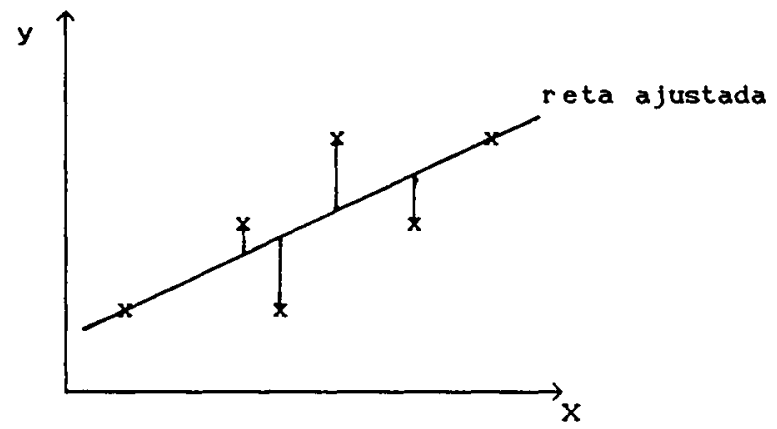

FIGURA 1. Solução referente à norma $L_{1}$

Neste caso, a solução obtida interpola os dados em pelo menos dois pontos, isto é, pelo menos duas equações são satisfeitas com igualdade.

- Norma $L_{2}:\|r(\alpha, \beta)\|_{2}=\left[\sum_{l=1}^{m}\left(r_{1}(\alpha, \beta)\right)^{2}\right]^{1 / 2}$.

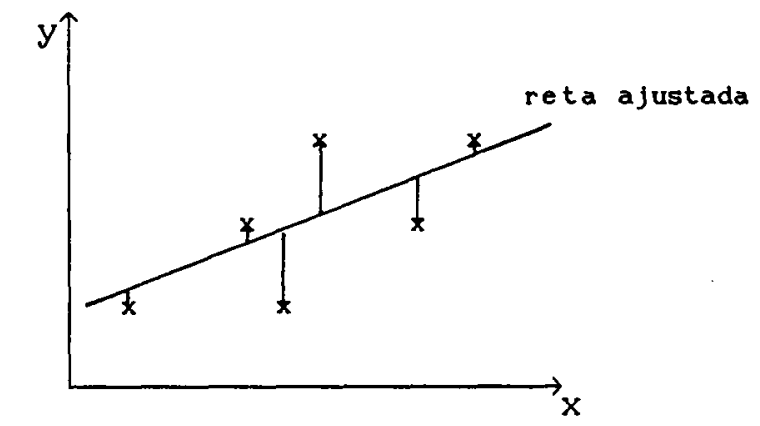

FIGURA 2. Solução referente à norma $\mathrm{L}_{2}$ 
Em geral, nenhuma equação é satisfeita.

- Norma $L_{\infty}:\|r(\alpha, \beta)\|_{\infty}=\max _{1}\left|r_{1}(\alpha, \beta)\right|$.

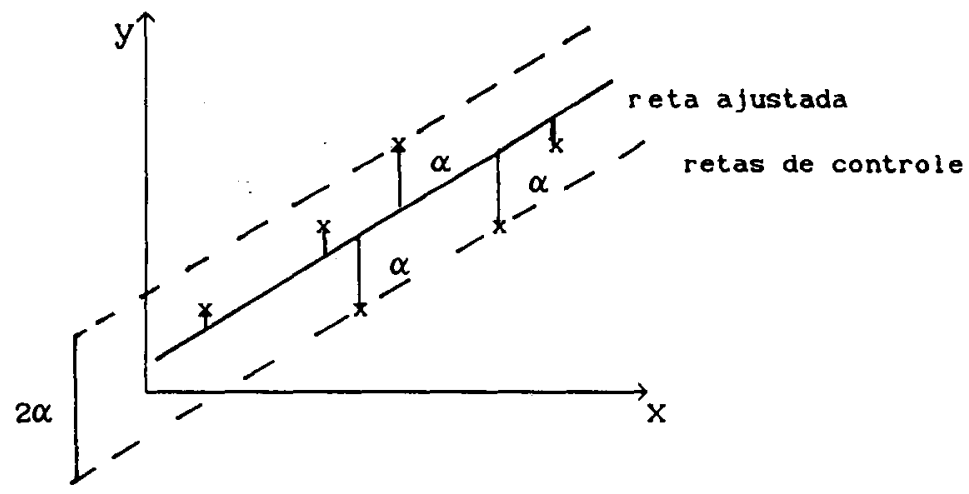

FIGURA 3. Solução referente à norma $L_{\infty}$

Este é o caso a ser estudado neste trabalho, em que os maiores resíduos devem ser mínimos. Os resíduos que tem o maior valor, denotados por $\alpha$, são pelo menos três. As normas anteriores admitiriam um resíduo grande, desde que a soma total fosse minimizada, influenciando-se pouco com pontos distoantes. A norma de Chebyshev, entretanto, é totalmente influenciada por todos os pontos. Observe que todos os pontos estão situados entre as duas retas paralelas tracejadas (retas de controle) na Fig. 3.

\subsection{Uma Aplicação na Metrologia.}

Um problema que surge na Metrologia (área da Engenharia Mecânica) é a determinação de Planicidade de Superfícies.

Dizemos que a PLANICIDADE de uma superfície é a menor distancia entre dois planos paralelos, denominados Planos de Controle, os quais contenham toda a superfície. 
Aproximamos a superfície por um plano, denominado Plano Médio - qual é paralelo aos Planos de Controle, como vemos na Fig. 4.

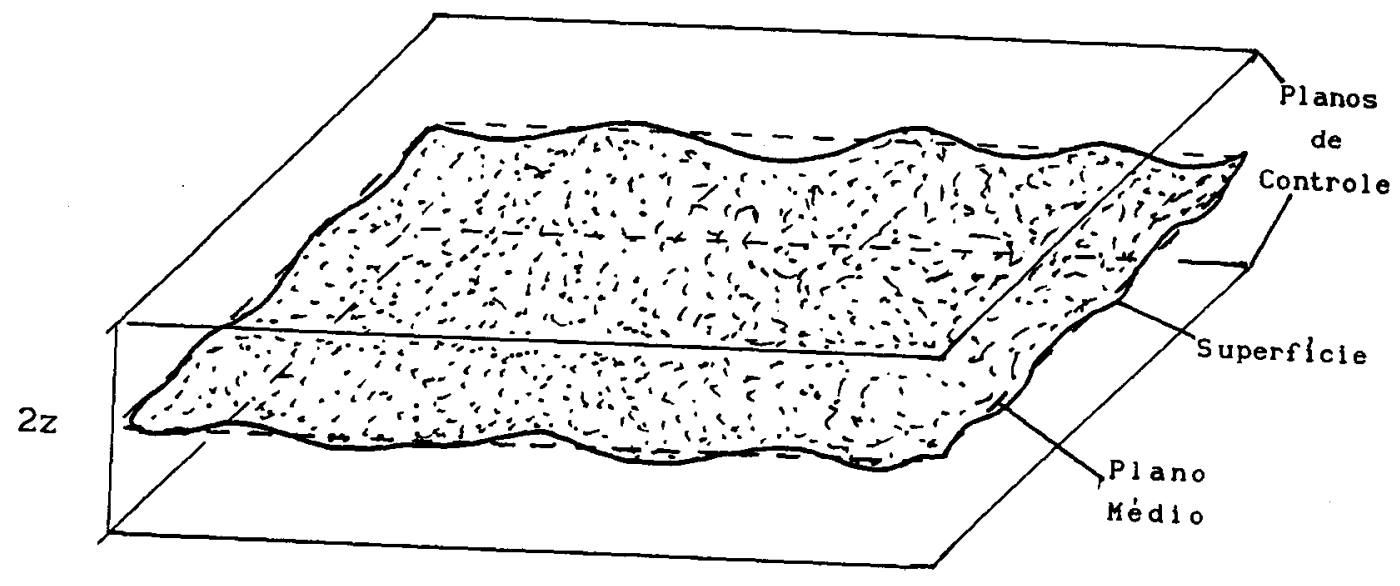

FIGURA 4. Representação de Planicidade

Para determinar a PLANICIDADE de uma superfície $(x, y, h)$, onde $h$ é a altura correspondente às coordenadas $(x, y)$, procedemos como segue. Definimos uma malha de pontos onde a superfície será medida, isto é, ao invés de termos $h=h(x, y)$, teremos, $h_{1}=h\left(x_{1}, y_{1}\right)$, (Fig.5).

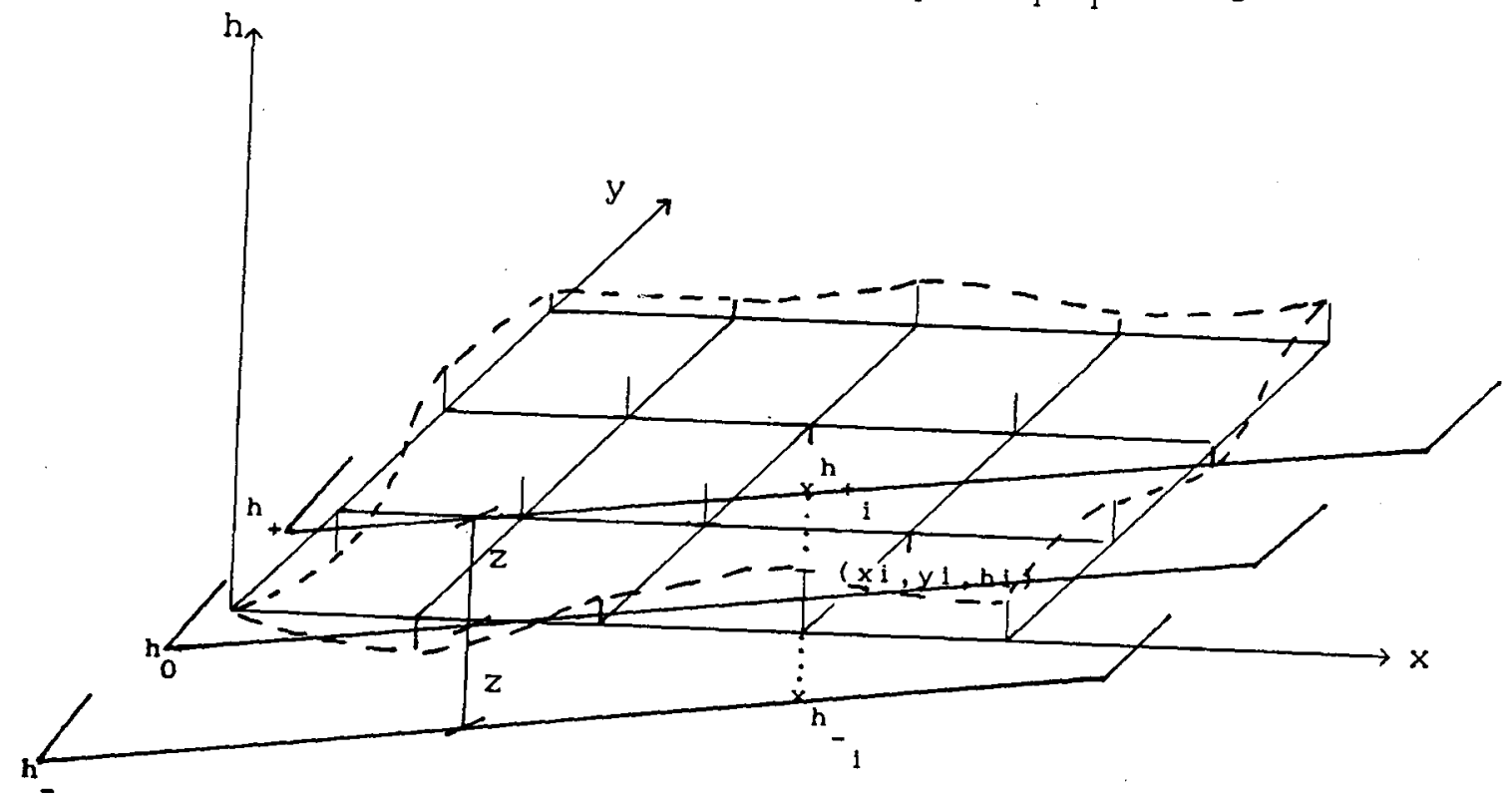

FIGURA 5. Discretização da superfície 
As equações dos planos que constam da Fig. 5 são dados por:

- Plano Médio:

$$
h_{0}=\varepsilon x+\beta y+\gamma
$$

- Planos de Controle:

$$
\begin{aligned}
& h_{+}=\varepsilon x+\beta y+\gamma+z \\
& h_{-}=\varepsilon x+\beta y+\gamma-z .
\end{aligned}
$$

Pela definição de Planos de Controle, temos:

$$
\begin{aligned}
& \mathrm{h}_{+}=\varepsilon \mathrm{x}_{1}+\beta \mathrm{y}_{1}+\gamma+\mathrm{z} \geq \mathrm{h}_{1} \\
& \mathrm{~h}_{-}=\varepsilon \mathrm{x}_{1}+\beta \mathrm{y}_{1}+\gamma-\mathrm{z} \leq \mathrm{h}_{1},
\end{aligned}
$$

ou seja,

$$
h_{1}-z \leq \varepsilon x_{1}+\beta y_{1}+\gamma \leq h_{i}+z, \quad 1 \leq i \leq m .
$$

Para quaisquer valores de $\alpha, \beta, \gamma$ e $z$ em (1.3), temos que a superfície está contida entre os Planos de Controle.

Queremos minimizar a distancia entre os planos de Controle, sujeito a (1.3), isto é queremos resolver o problema:

$$
\begin{aligned}
& \min z \\
& \text { s.a. } h_{1}-z \leq \varepsilon x_{1}+\beta y_{1}+\gamma \leq h_{1}+z \text {, }
\end{aligned}
$$


$\operatorname{com} 1 \leq i \leq m$.

Note que (1.3) pode ser escrita equivalentemente por:

$$
-z \leq \varepsilon x_{1}+\beta y_{1}+\gamma-h_{1} \leq z, \quad 1 \leq i \leq m,
$$

ou seja,

$$
\left|\varepsilon x_{1}+\beta y_{1}+\gamma-h_{1}\right| \leq z, \quad 1 \leq i \leq m .
$$

o problema (1.4) é, então, equivalente a:

$$
\min \left\{\max \left|\varepsilon x_{1}+\beta y_{1}+\gamma-h_{1}\right|, 1 \leq 1 \leq m\right\}
$$

ou, ainda,

$$
\text { Min }\|z\|_{\infty}
$$

s.a. $X \theta+z=h$

onde: $X=\left[\begin{array}{ccc}x_{1} & y_{1} & 1 \\ \vdots & \vdots & \vdots \\ x_{m} & y_{m} & 1\end{array}\right] \in \mathbb{R}^{m \times 3}, \theta=\left[\begin{array}{c}\varepsilon \\ \beta \\ \gamma\end{array}\right] \in \mathbb{R}^{3}, \quad h=\left[\begin{array}{c}h_{1} \\ \vdots \\ h_{m}\end{array}\right] \in \mathbb{R}^{m} e$ $z=\left[\begin{array}{l}z_{1} \\ \vdots \\ z_{m}\end{array}\right] \in \mathbb{R}^{m}$

Conforme apresentado em (1.2), (1.5) é o problema de determinar a solução de Chebyshev. Assim, mostramos que o problema de determinar a 
Planicidade de uma superfície consiste na resolução de um Sistema Linear Inconsistente, no senso da norma de Chebyshev. Observamos que o problema equivalente (1.4) é um problema de Programação Linear que será objeto de atenção no próximo capítulo.

A literatura existente sobre Planicidade, em sua quase totalidade faz uso do Método dos Mínimos Quadrados, por sua simplicidade. Entretanto, este método produz apenas uma solução aproximada para o problema de determinar Planicidade de superficies, como veremos no Capítulo 3. 
CAPITULO 2

\section{RESOLUC̣ÃO VIA PROGRAMAÇAOO LINEAR}

Neste Capítulo, abordamos o problema (1.2) do ponto de vista da Programação Linear.

2.1 Problema de Programação Linear Equivalente.

O problema (1.2) pode ser escrito por:

$$
\begin{aligned}
& \min \|r\|_{\infty} \\
& \text { s.a. } A x+r=b
\end{aligned}
$$

Definindo $z=\max \left|r_{1}\right|$, com $1 \leq i \leq m$, temos:

$$
\left|r_{1}\right| \leq \max \left|r_{1}\right|=z
$$

portanto, o problema (2.1) é equivalente ao seguinte problema de Programação Linear:

$$
\begin{gathered}
\min z \\
\text { s.a. }-z \leq r_{1} \leq z,
\end{gathered}
$$

$\operatorname{com} 1 \leq i \leq m$, 
ou seja,

$$
\text { min } z=b-A x \leq z e
$$

ou ainda,

$$
\left.\min \quad \begin{array}{cc}
z & e \\
-A & e
\end{array}\right]\left[\begin{array}{l}
x \\
z
\end{array}\right] \geq\left(\begin{array}{c}
b \\
-b
\end{array}\right)
$$

onde $e^{t}=(1, \ldots, 1) \in \mathbb{R}^{m}$.

o problema (2.2) pode ser resolvido por métodos de Programação Linear, como, por exemplo, o método Simplex.

\subsection{Problema Dual.}

Ao problema (2.1) temos associado o seguinte problema dual [ 3]:

$$
\begin{aligned}
& \max \lambda^{t} b \\
& \text { s. a. } A^{t} \lambda=0 \\
& \|\lambda\|_{1}=1
\end{aligned}
$$

onde $\lambda \in \mathbb{R}^{\mathfrak{m}}$, o qual é um problema com restrições lineares por partes.

Podemos linearizar (2.3), fazendo $\lambda=\lambda^{+}-\lambda^{-}$, onde $\lambda^{+} \geq 0, \lambda^{-} \geq 0$, obtendo o seguinte problema de Programação Linear: 


$$
\begin{array}{ll}
\max & \left(\lambda^{+}\right)^{t} b-\left(\lambda^{-}\right)^{t} b \\
\text { s.a. } & A^{t} \lambda^{+}-A^{t} \lambda^{-}=0 \\
& \sum_{1=1}^{m} \lambda_{1}^{+}+\sum_{1=1}^{m} \lambda_{1}^{-}=1 \\
& \lambda^{+} \geq 0, \lambda^{-} \geq 0
\end{array}
$$

O problema (2.4) pode também ser obtido tomando-se 0 dual de (2.2) [3].

Neste trabalho, aplicamos uma especialização do método Simplex ao problema (2.4). Observamos que, apesar de trabalharmos com o problema linearizado, somente a matriz $A^{t}$ e $O$ vetor $b$ precisam ser armazenados ao aplicarmos esta especialização do método SIMPLEX, pois trabalhamos com um sinal para identificarmos a variável $\lambda_{1}^{+}$ou $\lambda_{1}^{-}$.

Os passos da especialização do método Simplex aplicado especificamente ao problema de Planicidade, bem como seu algoritmo, serão detalhados no Capítulo 3. 


\section{CAPITULO 3}

\section{ESTUDO DE CASO: PLANICIDADE DE SUPERFICIES}

Neste Capítulo, detalhamos os passos da especialização do método Simplex para o caso de Planicidade de Superfícies e desenvolvemos um algoritmo.

Fazendo uso da mesma notação utilizada no Capítulo 1, seção 1.2 , ao problema (1.5) temos associado o seguinte problema dual:

$$
\begin{array}{ll}
\max & \lambda^{t} h \\
\text { s.a. } & x^{t} \lambda=0 \\
& \|\lambda\|_{1}=1
\end{array}
$$

Este problema pode ser linearizado como (2.4), portanto, temos:

$$
\begin{array}{ll}
\max & \left(\lambda^{+}\right)^{\mathrm{t}} h-\left(\lambda^{-}\right)^{\mathrm{t}} \mathrm{h} \\
\text { s.a. } & \mathrm{x}^{\mathrm{t}} \lambda^{+}-\mathrm{x}^{\mathrm{t}} \lambda^{-}=0 \\
& \sum_{i=1}^{\mathrm{m}} \lambda_{i}^{+}+\sum_{i=1}^{\mathrm{m}} \lambda_{1}^{-}=1 \\
& \lambda^{+} \geq 0, \lambda^{-} \geq 0
\end{array}
$$

Toda teoria desenvolvida neste Capítulo, bem como o algoritmo, tem por objetivo resolver o problema (3.1).

\subsection{Estrutura de Dados.}


Observamos nesta seção que, devido à estrutura de dados do problema não será necessário armazenarmos a matriz $X$, e fornecemos um procedimento para gerarmos as suas linhas.

Como vimos no Capítulo 1, seção 1.2., ao fazermos a discretização de uma superfície, a cada ponto $\left(x_{i}, y_{j}\right)$ corresponde uma cota $h_{k}$. Os pontos $\left(x_{1}, y_{j}\right)$ são obtidos da seguinte maneira:

$$
\begin{gathered}
x_{0}=0, y_{0}=0, \\
x_{i+1}=x_{i}+\Delta x, i=0,1, \ldots, M_{h}-1
\end{gathered}
$$

e

$$
y_{j+1}=y_{j}+\Delta y, j=0,1, \ldots, M_{v}-1
$$

onde: $M_{h} \in \mathbb{N}$ é 0 número de medidas feitas na horizontal; $M_{v} \in \mathbb{N}$ é 0 número de medidas feitas na vertical; $k=1, \ldots,\left(M_{h} * M_{v}\right)$, sendo $\left(M_{h}^{*} M_{v}\right) \circ$ número total de pares $\left(x_{i}, y_{j}\right),($ Fig. 6).

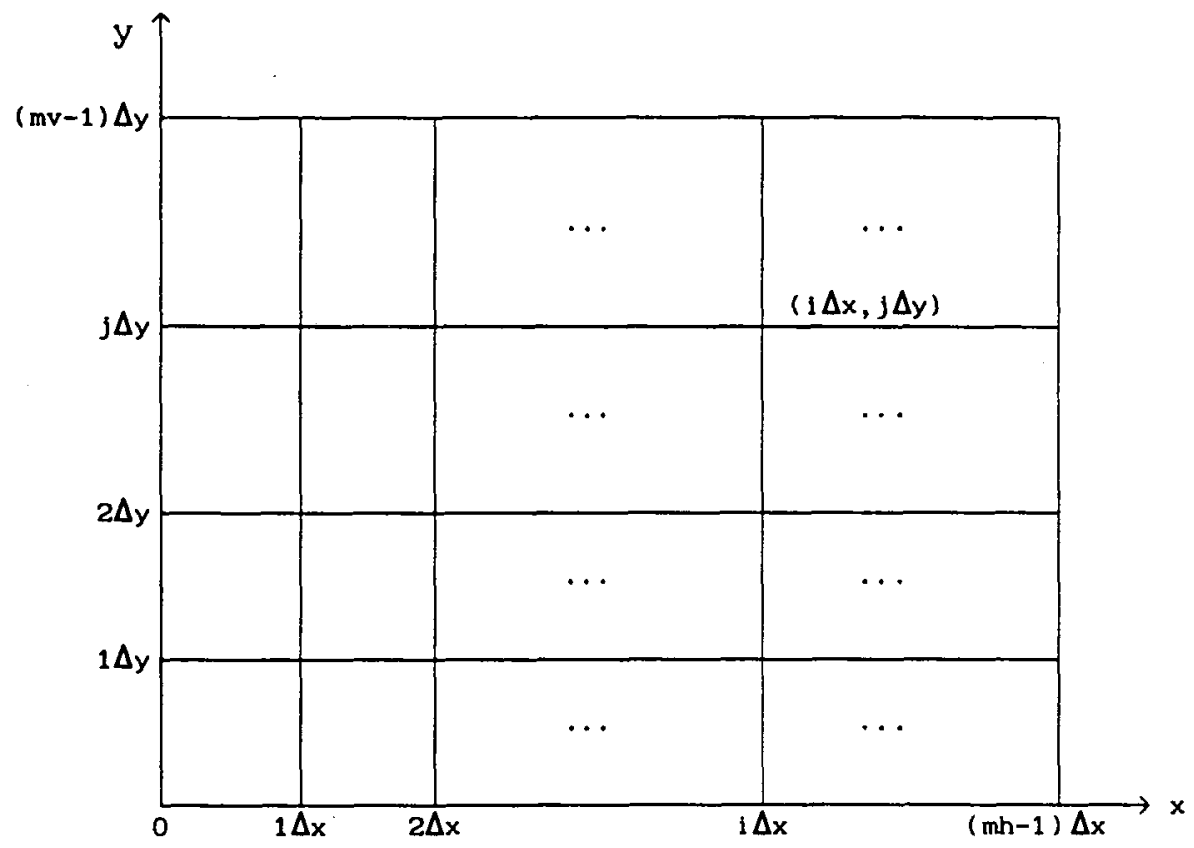

FIGURA 6. Discretização da superfície em termos de $\Delta x$ e $\Delta y$ 
Usando uma nova escala, podemos associar cada nó (i $\Delta x, j \Delta y$ ) a um nó $(i, j)$, com $0 \leq i \leq\left(M_{h}-1\right)$ e $0 \leq j \leq\left(M_{v}-1\right)$. Obtemos assim a Fig. 7 .

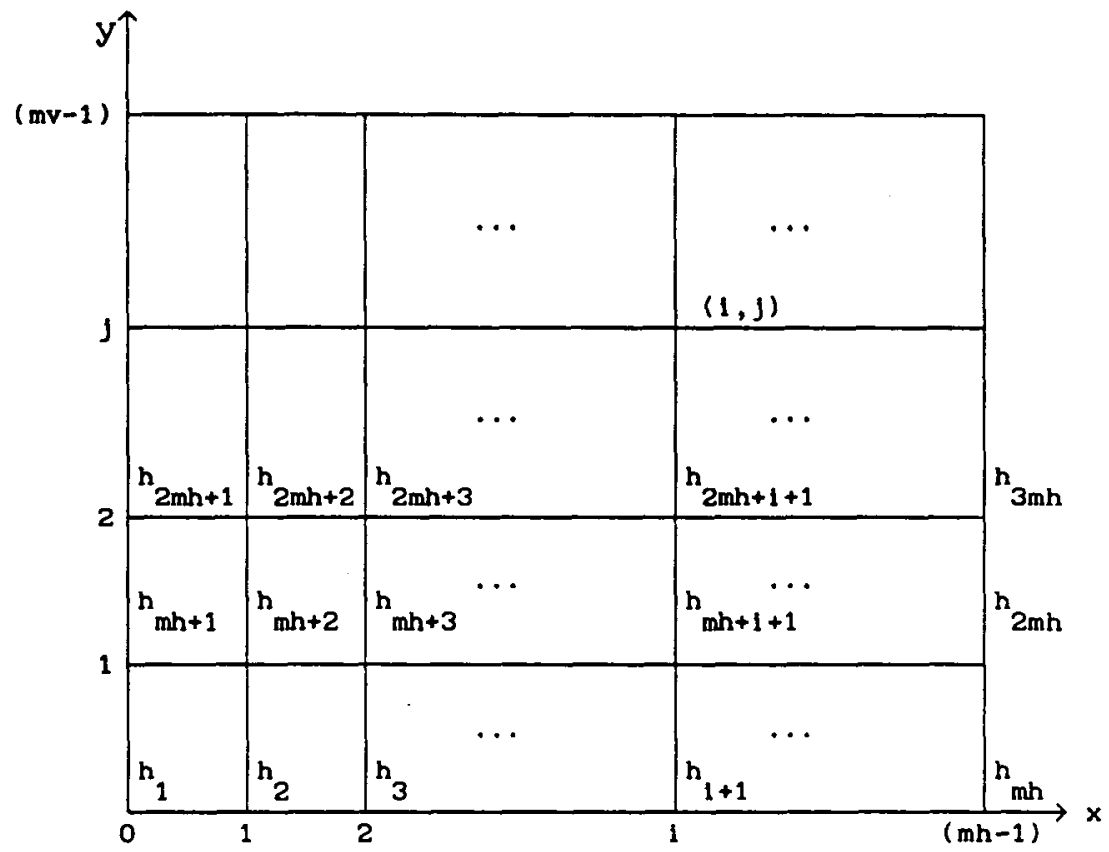

FIGURA 7. Mudança de escala da discretização

Observando que a matriz $X$ em (1.5) tem uma estrutura especifica, pois cada linha está associada a um nó $(i, j)$, optamos por não armazenar esta matriz. Suas linhas (ou colunas de $x^{t}$ ) serão geradas sempre que necessário. O vetor das medições $h$ é armazenado conforme Fig. 7.

Sabemos que a cada nó $(i, j)$ corresponde o indice $k$ de $h$, com $1 \leq k \leq\left(M_{h}^{*} M_{v}\right)$, ou seja:

$$
(i, j) \longrightarrow k=\left(j * M_{h}\right)+i+1
$$

Queremos resolver o problema inverso: "Dado k, como determinarmos 
i e $j$ ?". Isto pode ser obtido do seguinte modo:

$$
\frac{k-1}{M_{h}}=j+\frac{i}{M_{h}}
$$

ou seja, $j$ é o quociente inteiro da divisão de $k-1$ por $M_{h}$ e i é o resto inteiro dessa divisão.

Para distinguirmos as colunas de $x^{t}$ e de $-x^{t}$ usamos um sinal $s$, que será igual a 1 , se a coluna pertencer a $x^{t}$, e -1 , se a coluna pertencer a $-\mathbf{x}^{\mathrm{t}}$.

Na seção seguinte, precisaremos selecionar três colunas linearmente independentes da matriz $x^{t}$ para determinarmos a solução inicial do problema. Conforme podemos observar na Fig. 7 , se os pontos não estão alinhados, os vetores colunas correspondentes na matriz $\mathbf{x}^{\mathrm{t}}$ são linearmente independentes [10]. Portanto, o procedimento adotado será o de escolher colunas que correspondam aos nós que não sejam colineares.

\subsection{Solução Básica Inicial.}

Para aplicarmos a especialização do Método Simplex, precisamos ter disponível uma solução básica inicial. Nesta seção fornecemos um procedimento para a determinação da solução básica inicial, para isso, trabalhamos, primeiramente, com o conjunto de restrições dado por:

$$
x^{t} \lambda^{+}-x^{t} \lambda^{-}=0
$$

e depois nos preocupamos com a restrição de normalização do vetor.

Selecionamos três colunas linearmente independentes da matriz 
$\mathbf{X}^{t} \in \mathbb{R}^{3 \times m}$, e uma quarta coluna arbitrariamente, todas associadas aos maiores valores absolutos dos $h_{1}$ 's. Denominamos $B^{\prime}$ a submatriz de $x^{t}$ composta por estas quatro colunas, tal que, $B^{\prime} \in \mathbb{R}^{3 \times 4}$. 0 conjunto dos indices relacionados a estas colunas é chamado de conjunto de índices básicos, onde $B_{i}$ denota $O i$-ésimo indice básico, com $i=1, \ldots, 4$ e $\circ$ conjunto dos indices restantes é o conjunto dos índices não básicos,

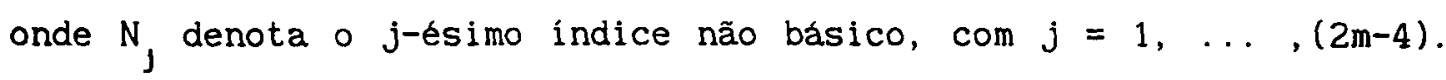
Associamos, inicialmente, a cada coluna de $B^{\prime}$ um sinal $s_{1}$, tal que, $s_{1}=1$, para $i=1, \ldots, 4$, pois as colunas pertencem a $x^{t}$.

Portanto, B' pode ser escrita na forma:

$$
B^{\prime}=\left[\begin{array}{llll}
s_{1} u_{B} & s_{2} u_{B_{2}} & s_{3} u_{B} & s_{4} u_{B}
\end{array}\right]
$$

onde, $u_{B_{1}}$ é a $B_{1}$-ésima coluna da matriz $x^{t}$ e $s_{1}$ é o sinal associado a a ela, para $1 \leq i \leq 4$.

Anulamos as $(2 m-4)$ variáveis que não estão associadas ao conjunto de índices básicos e resolvemos o seguinte sistema:

$$
B^{\prime} \lambda_{B}^{\prime}=0 \text {, }
$$

onde $\lambda_{B}^{\prime t}=\left(\lambda_{B_{1}}^{\prime}, \lambda_{B_{2}}^{\prime}, \lambda_{B_{3}}^{\prime}, \lambda_{B_{4}}^{\prime}\right) \in \mathbb{R}^{4}$

Como o número de variáveis livres do sistema (3.2) é igual a 1, atribuímos à variável $\lambda_{B_{4}}^{\prime} \circ$ valor 1 ; este valor será alterado posteriormente, pois a restrição de normalização ainda não foi 
satisfeita. Determinamos, assim, uma solução particular para o sistema, podendo ocorrer duas situações:

SITUAÇÃO 1: $\quad \lambda_{B}^{\prime} \geq 0$.

Se todas as coordenadas do vetor $\lambda_{B}^{\prime}$ são não negativas, normalizamos este vetor para satisfazer a última restrição, obtendo:

$$
\lambda_{B}=\frac{\lambda_{B}^{\prime}}{\left\|\lambda_{B}^{\prime}\right\|}
$$

Deste modo, $\lambda_{B}$ é solução para o seguinte sistema:

$$
B \lambda_{B}=\left(\begin{array}{l}
0 \\
0 \\
0 \\
1
\end{array}\right) \text {, }
$$

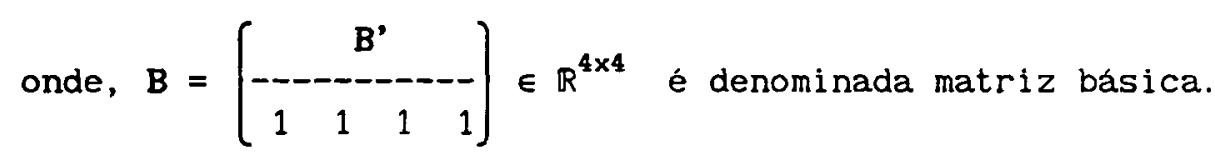

SITUAÇÃO 2: $\lambda_{B_{1}^{\prime}}^{\prime}<0$, para algum i.

Caso algum $\lambda_{B_{i}}^{\prime}$ seja negativo, para algum $i, 1 \leq i \leq 3$ (pois $\left.\lambda_{B_{4}^{\prime}}^{\prime}=1\right)$, redefinimos a matriz $B^{\prime}$, fazendo uma troca de variável, ou seja, trocando a $B_{i}$-ésima coluna de $x^{t}$ pela $B_{i}$-ésima coluna $-x^{t}$, $1 \leq i \leq 3$. Isto equivale a dizer que será trocado o sinal $s_{i}$, isto é, $s_{1}=-1$. Consequentemente, a solução de (3.2) será alterada, e $\lambda_{B_{1}^{\prime}}^{\prime}$ será dado por $-\lambda_{B_{1}^{\prime}}^{\prime}$, sendo desnecessária a resolução do novo sistema, 
pois de (3.2), temos:

$$
B^{\prime} \lambda_{B}^{\prime}=0,
$$

ou seja,

$$
s_{1} u_{B_{1}} \lambda_{B_{1}}^{\prime}+s_{2} u_{B_{2}} \lambda_{B_{2}}^{\prime}+s_{3} u_{B_{3}} \lambda_{B_{3}}^{\prime}+s_{4} u_{B_{4}} \lambda_{B_{4}}^{\prime}=0
$$

ou, ainda, supondo $\lambda_{B}^{\prime}<0$, trocamos a primeira coluna, isto é, redefinimos B', como segue:

$$
-s_{1} u_{B_{1}} \bar{\lambda}_{B_{1}}^{\prime}+s_{2} u_{B_{2}} \bar{\lambda}_{B_{2}}^{\prime}+s_{3} u_{B_{3}} \bar{\lambda}_{B_{3}}^{\prime}+s_{4} u_{B_{4}} \bar{\lambda}_{B_{4}}^{\prime}=0
$$

onde, $\bar{\lambda}_{B}^{\prime t}=\left(\bar{\lambda}_{B_{1}}^{,}, \bar{\lambda}_{B_{2}}^{\prime}, \bar{\lambda}_{B_{3}}^{\prime}, \bar{\lambda}_{B_{4}}^{\prime}\right) \in \mathbb{R}^{4}$ é a nova solução.

Reescrevendo (3.5), da seguinte forma:

$$
s_{1} u_{B_{1}}\left(-\bar{\lambda}_{B_{1}}^{\prime}\right)+s_{2} u_{B_{2}} \bar{\lambda}_{B_{2}}^{\prime}+s_{3} u_{B_{3}} \bar{\lambda}_{B_{3}}^{\prime}+s_{4} u_{B_{4}} \bar{\lambda}_{B_{4}}^{\prime}=0,
$$

e comparando com (3.4), concluímos:

$$
\bar{\lambda}_{B_{1}}^{\prime}=-\lambda_{B_{1}}^{\prime}, \bar{\lambda}_{B_{2}}^{\prime}=\lambda_{B_{2}}^{\prime}, \bar{\lambda}_{B_{3}}^{\prime}=\lambda_{B_{3}}^{\prime} \text { e } \bar{\lambda}_{B_{4}}^{\prime}=\lambda_{B_{4}}^{\prime} .
$$

Procedemos, agora, com a normalização do vetor, como na situação 1.

\subsubsection{Posto de B.}

Nesta seção provaremos que a matriz B, fornecida poe (3.3), é uma matriz básica.

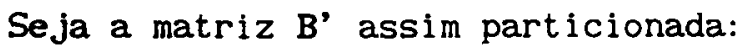




$$
B^{\prime}=\left[\begin{array}{l:ll}
B_{1} & S_{4} U_{B_{4}}
\end{array}\right]
$$

A submatriz $B_{1}$ é dada por $B_{1}=\left[\begin{array}{llll}s_{1} u_{B_{1}} & s_{2} u_{B_{2}} & s_{3} u_{B_{3}}\end{array}\right] \in \mathbb{R}^{3 \times 3}$ e posto $\left(B_{1}\right)=3$, conforme construção efetuadas na seção 3.2 .

Então, a matriz B em (3.2) pode ser reescrita da seguinte forma:

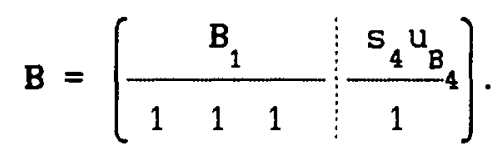

Como posto $\left(B_{1}\right)=3$, concluímos que $B_{1}$ é inversível. Aplicando Eliminação de Gauss em bloco à matriz (3.6), com o objetivo de zerar a última linha temos :

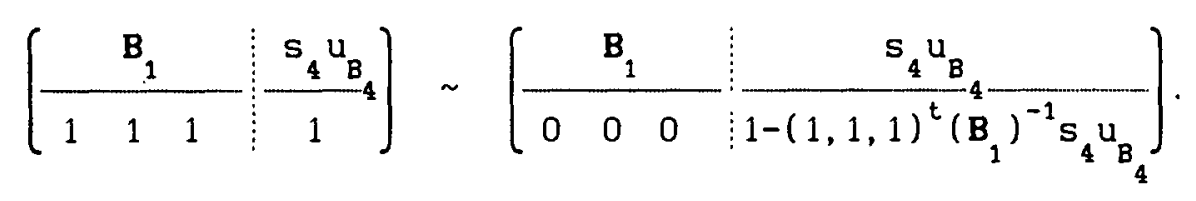

Sabemos, por construção, que:

$$
\left[\begin{array}{c}
\lambda_{B_{1}} \\
\lambda_{B_{2}} \\
\lambda_{B_{3}}
\end{array}\right]=-\left(B_{1}\right)^{-1} s_{4} u_{B_{4}} \geq 0
$$

então,

$$
1-(1,1,1)^{t}\left(B_{1}\right)^{-1} s_{4} u_{B_{4}} \geq 1
$$


Concluímos que a matriz B tem posto igual a 4 .

Observamos que a estratégia de selecionarmos as 4 colunas, como visto na seção 3.2 , como sendo aquelas associadas aos maiores valores absolutos dos $h_{1 ' s}$ ' também foi usada em [12] e tem por objetivo reduzir o número de iterações, bem como o tempo computacional. Fizemos várias experiéncias computacionais e concluimos que esta prática realmente nos fornece um tempo computacional melhor do que a escolha ao acaso.

\subsection{Vetor Multiplicador (ou Solução Primal).}

Ao calcularmos o vetor multiplicador, observamos que este nos fornece o valor das variáveis primais e da função objetivo primal. Portanto, nesta seção mostramos como obter estes valores.

Para determinarmos o valor das variáveis primais e da função objetivo primal, resolvemos o seguinte sistema:

$$
B^{t} \Pi=h_{B}
$$

onde: $B^{t}=\left[\begin{array}{c:c}B_{1}^{t} & 1 \\ \ldots \ldots & 1 \\ \hdashline s_{4} u^{t} & 1\end{array}\right] \in \mathbb{R}^{4 \times 4} ; h_{B}^{t}=\left[s_{1} h_{B_{1}}, s_{2} h_{B_{2}}, s_{3} h_{B_{3}}, s_{4} h_{B_{4}}\right] \in \mathbb{R}^{4}$ e $\Pi^{t}=\left[\begin{array}{ll}\theta^{t} & \alpha\end{array}\right] \in \mathbb{R}^{4}$. Aqui $\theta \in \mathbb{R}^{3}$ é o vetor das variáveis primais e $\alpha \in \mathbb{R}$, o valor da função objetivo primal, pois de (3.7), temos:

$$
s_{1} u_{B_{1}}^{t} \theta+\alpha=s_{1} h_{B_{1}}, \quad 1 \leq i \leq 4,
$$


ou, multiplicando por $s_{i}$,

$$
u_{B_{1}}^{t} \theta+z_{B_{i}}=h_{B_{1}}, \quad 1 \leq i \leq 4,
$$

com $z_{B_{1}}=s_{1} \alpha$. Temos que (3.8) corresponde às equações primais associadas aos indices básicos.

Se $\alpha \geq 0$, então representamos $\alpha$ por:

$$
\alpha=\left\|z_{B}\right\|_{\infty}=\left|z_{B_{1}}\right| \text {. }
$$

Caso aconteça $\alpha<0$ durante a aplicação do método Simplex, o nosso procedimento será o seguinte:

- Trocamos o valor da variável $\alpha$, isto é $\alpha$ será dado por $-\alpha$, tornando esse valor positivo e, para continuar valendo a igualdade no sistema (3.5), s receberá -s, ou seja:

$$
\left[\begin{array}{c}
-s_{1} u_{B_{1}}^{t} \\
-s_{2} u_{B_{2}}^{t} \\
-s_{3} u_{B_{3}}^{t} \\
-s_{4} u_{B_{4}}^{t}
\end{array}\right] \theta+\left[\begin{array}{l}
1 \\
1 \\
1 \\
1
\end{array}\right] \quad(-\alpha)=\left(\begin{array}{l}
-s_{1} h_{B_{1}} \\
-s_{2} h_{B_{2}} \\
-s_{3} h_{B_{3}} \\
-s_{4} h_{B}
\end{array}\right]
$$

\subsection{Custos Relativos.}

o critério de parada para o nosso algoritmo é fornecido pelo 
valor dos custos relativos, ou seja, são eles que nos indicam se a solução é otima ou não. Nesta seção, apresentamos um procedimento para o calculo dos mesmos.

Utilizando a estrutura do problema (3.1) temos que calcular os custos relativos para as variáveis não básicas $\lambda^{+}$e $\lambda^{-}$.

Para as variáveis $\lambda^{+}$, temos:

$$
\hat{h}_{N_{j}}^{+}=h_{N_{j}}-\theta^{t}\left[\begin{array}{l}
x_{N_{j}} \\
y_{N_{j}} \\
1
\end{array}\right]-\alpha,
$$

$\operatorname{com} 1 \leq j \leq(m-4)$.

Para as variáveis $\lambda^{-}$, temos:

$$
\hat{h}_{N_{j}}^{-}=-h_{N_{j}}+\theta^{t}\left[\begin{array}{l}
x_{N_{j}} \\
y_{N_{j}} \\
1
\end{array}\right]-\alpha=-\left[h_{N_{j}}-\theta^{t}\left[\begin{array}{c}
x_{N_{j}} \\
y_{N_{j}} \\
1
\end{array}\right]\right)-\alpha,
$$

$\operatorname{com} 1 \leq j \leq(m-4)$

$$
\begin{gathered}
\text { Chamando AUX = } h_{N_{j}}-\theta^{t}\left[\begin{array}{c}
x_{N_{j}} \\
y_{N_{j}} \\
1
\end{array}\right],(3.9) \text { e (3.10) ficam: } \\
\hat{h}_{N_{j}^{+}}^{+}=A U X-\alpha, \\
\hat{h}_{N_{j}^{-}}^{-}=-A U X-\alpha .
\end{gathered}
$$

Para que a factibilidade não seja violada, devemos ter: 


$$
\hat{\mathrm{h}}_{\mathrm{N}_{j}}^{+} \leq 0 \text { e } \hat{\mathrm{h}}_{\mathrm{N}_{j}}^{-} \leq 0
$$

com $1 \leq j \leq(m-4)$, ou seja,

$$
-\alpha \leq \mathrm{AUX} \leq \alpha .
$$

Caso a factibilidade seja violada, isto é, caso ocorra:

$$
A U X>\alpha \text { ou } A U X<-\alpha \text {, }
$$

devemos fazer a variável que mais viola a factibilidade fazer parte da nova base.

\subsubsection{Observação.}

Se $\lambda_{B_{1}^{+}}^{+}$(ou $\lambda_{B_{1}^{-}}^{-}$), para qualquer $i$, fizer parte da base, mostraremos que $\circ$ custo relativo associado a $\lambda_{B_{1}}^{-}$(ou $\lambda_{B_{i}^{+}}^{+}$) é não positivo, e portanto não é candidato a entrar na base. Temos, então, as situações:

1) A variável $\lambda_{B_{i}^{+}}^{+}$é básica.

Calculando o custo relativo associado a $\lambda_{B_{1}^{+}}^{+}$, temos:

$$
\hat{h}_{B_{1}^{+}}^{+}=h_{B_{1}}-\theta^{t}\left[\begin{array}{c}
x_{B_{1}} \\
y_{B_{1}} \\
1
\end{array}\right]-\alpha=0,
$$


ou, equival entemente,

$$
h_{B_{1}}-\theta^{t}\left(\begin{array}{c}
x_{B} \\
y_{B_{1}} \\
1
\end{array}\right)=\alpha .
$$

Calculando o custo relativo associado $\lambda_{B_{1}^{-}}^{-}$, temos:

$$
\hat{h}_{B_{1}}^{-}=-\left[h_{B_{1}}-\theta^{t}\left[\begin{array}{c}
x_{B_{1}} \\
y_{B_{1}} \\
1
\end{array}\right]\right)-\alpha,
$$

de onde segue, por (3.11), que:

$$
\hat{\mathrm{h}}_{\mathrm{B}_{1}}^{-}=-\alpha-\alpha=-2 \alpha \leq 0 \text {, }
$$

pois $\alpha \geq 0$.

2) A variável $\lambda_{B_{1}^{-}}^{-}$é básica.

Calculando o custo relativo associado a $\lambda_{B_{1}^{-}}^{-}$:

$$
\hat{h}_{B_{1}}^{-}=-\left[h_{B_{1}}-\theta^{t}\left[\begin{array}{c}
x_{B_{1}} \\
y_{B_{1}} \\
1
\end{array}\right]-\alpha=0,\right.
$$

ou, equivalentemente, 


$$
h_{B_{1}}-\theta^{t}\left[\begin{array}{c}
x_{B_{1}} \\
y_{B_{i}} \\
1
\end{array}\right]=-\alpha .
$$

Calculando o custo relativo associado a $\lambda_{B_{1}^{+}}^{+}$:

$$
\hat{h}_{B_{1}^{+}}^{+}=h_{B_{1}}-\theta^{t}\left[\begin{array}{c}
x_{B_{1}} \\
y_{B_{1}} \\
1
\end{array}\right]-\alpha,
$$

usando (3.12),

$$
\hat{\mathrm{h}}_{B_{1}^{+}}^{+}=-\alpha-\alpha=-2 \alpha \leq 0,
$$

pois $\alpha \geq 0$.

Portando calculamos somente o custo relativo das variáveis não básicas $\lambda^{+}$e $\lambda^{-}$.

\subsection{Algoritmo Simplex Aplicado ao Problema de Planicidade.}

- algoritmo apresentado é uma especialização do algoritmo Simplex. Levamos em consideração que o problema sempre tem solução otima pois o zero é um limitante inferior para o problema. 


\section{Início}

- Determinação de uma solução básica inicial conforme seção 3.2

. PARE \& FALSO

. ENQUANTO ( NÃO PARE ) faça

Inicio

\{ solução básica corrente \}

.Seja B a matriz básica e a solução básica factível associada, dada por:

$$
\text { B } \lambda_{B}=\left(\begin{array}{l}
0 \\
0 \\
0 \\
1
\end{array}\right)
$$

\{ verificação da otimalidade \}

-i) Cálculo do vetor multiplicador (solução Primal)

$$
B^{t} \Pi=h_{B}
$$

-ii) Cálculo dos custos relativos

$$
\begin{aligned}
\text { Para } 1 & \leq j \leq(m-4), \\
\hat{h}_{N_{j}} & =h_{N_{j}}-\pi^{t} u_{N_{j}},
\end{aligned}
$$

-iii) Teste de Otimalidade

$\mathrm{Se}$

$$
\hat{h}_{N_{k}}=\operatorname{Max}\left\{\hat{h}_{N_{j}}^{+} \text {e } \hat{h}_{N_{j}}^{-}, 1 \leq j \leq(m-4)\right\} \leq 0
$$

A solução corrente é ótima. PARE $\leftarrow$ VERDADE.

Senão 


$$
s_{k}=\left\{\begin{aligned}
1, & \text { se } \hat{h}_{N_{k}}=\hat{h}_{N_{j}^{+}}^{+} \\
-1, & \text { se } \hat{h}_{N_{k}}=\hat{h}_{N_{j}}^{-}
\end{aligned}\right.
$$

Calcule a direção

$$
B d=s_{k} u_{k}
$$

$\{$ Determinação do Passo \}

$$
\varepsilon=\frac{\lambda_{B_{1}}}{d_{1}}=\min \left\{\frac{\lambda_{B_{1}}}{d_{1}}, d_{1}>0,1 \leq i \leq 4\right\}
$$

\section{\{ Atualização \}}

-Nova partição básica ( troque a l-ésima coluna de $B$ por $s_{k} u_{k}$, onde $u_{N_{k}}$ é a $N_{k}$-ésima coluna de $x^{t}$ )

Fim.

Fim.

\subsection{Experiência Computacional}

O algoritmo apresentado na seção 3.5 foi implementado utilizando a linguagem Pascal. Alguns exemplos foram executados num microcomputador padrão PC - 486, sem coprocessador matemático.

Nos exemplos 1 a 7 , aplicamos a especialização do método Simplex e comparamos a solução obtida com a dos Mínimos Quadrados, bem como o tempo computacional gasto. Os resultados computacionais são dados sob a forma de tabelas. 


\section{EXEMPLO 1:}

Os dados fornecidos neste exemplo foram extraidos de [11], os quais foram obtidos através da medição de um desempeno.

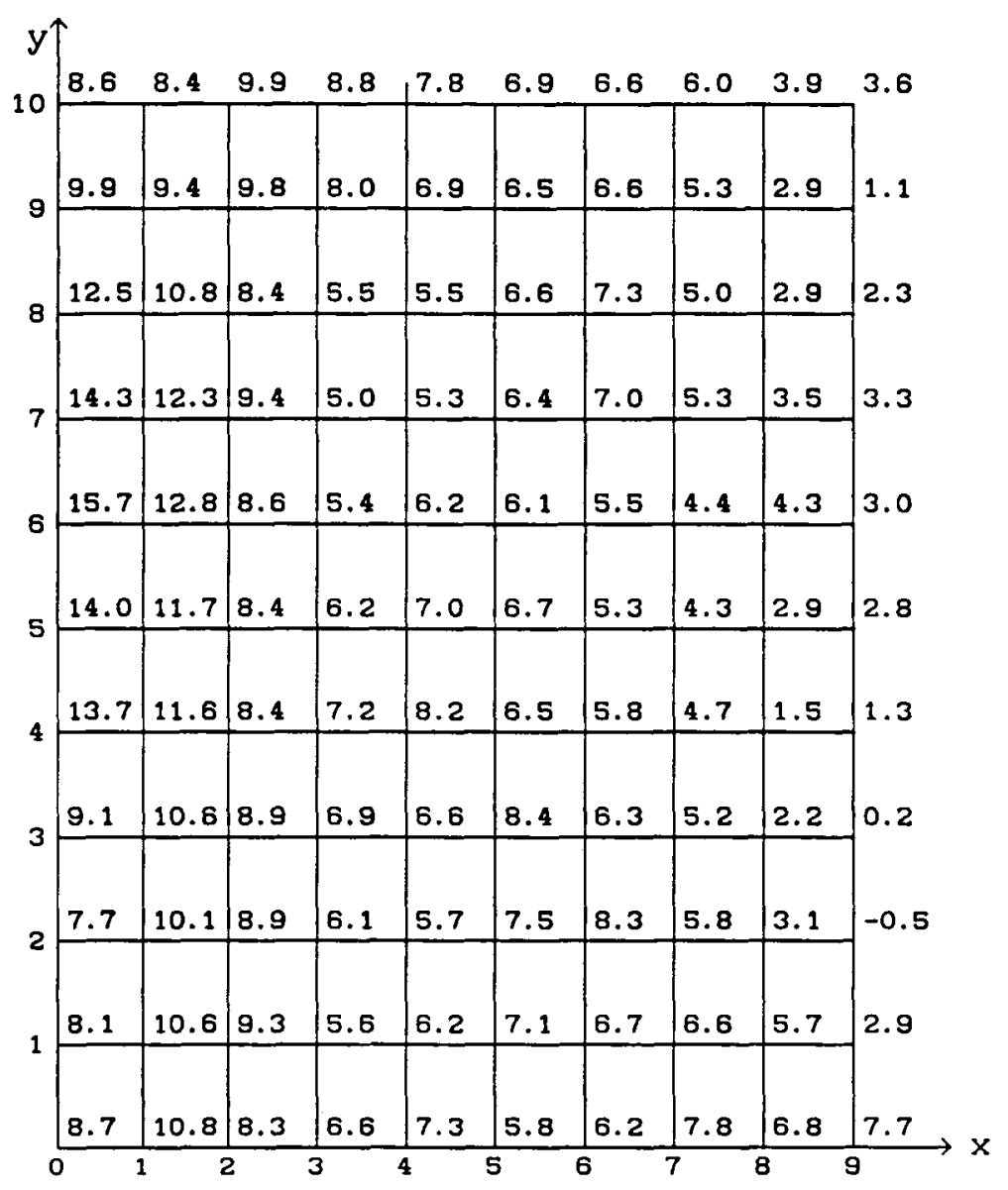

\begin{tabular}{|c|c|c|}
\cline { 2 - 3 } \multicolumn{1}{c|}{} & Solução $(\mu \mathrm{m})$ & $\begin{array}{c}\text { Tempo } \\
\text { Computacional (cs) }\end{array}$ \\
\hline Esp. Metodo Simplex & 8.13 & 82 \\
\hline Mínimos Quadrados & 8.32 & 39 \\
\hline
\end{tabular}


Para verificarmos quais alterações poderiam ocorrer nas soluções dos dois métodos fizemos algumas alterações no exemplo acima resultando nos exemplos 2,3 e 4 .

\section{EXEMPLO 2:}

Neste exemplo, alteramos para valores maiores as medições feitas no lado direito, nas últimas duas colunas, como segue:

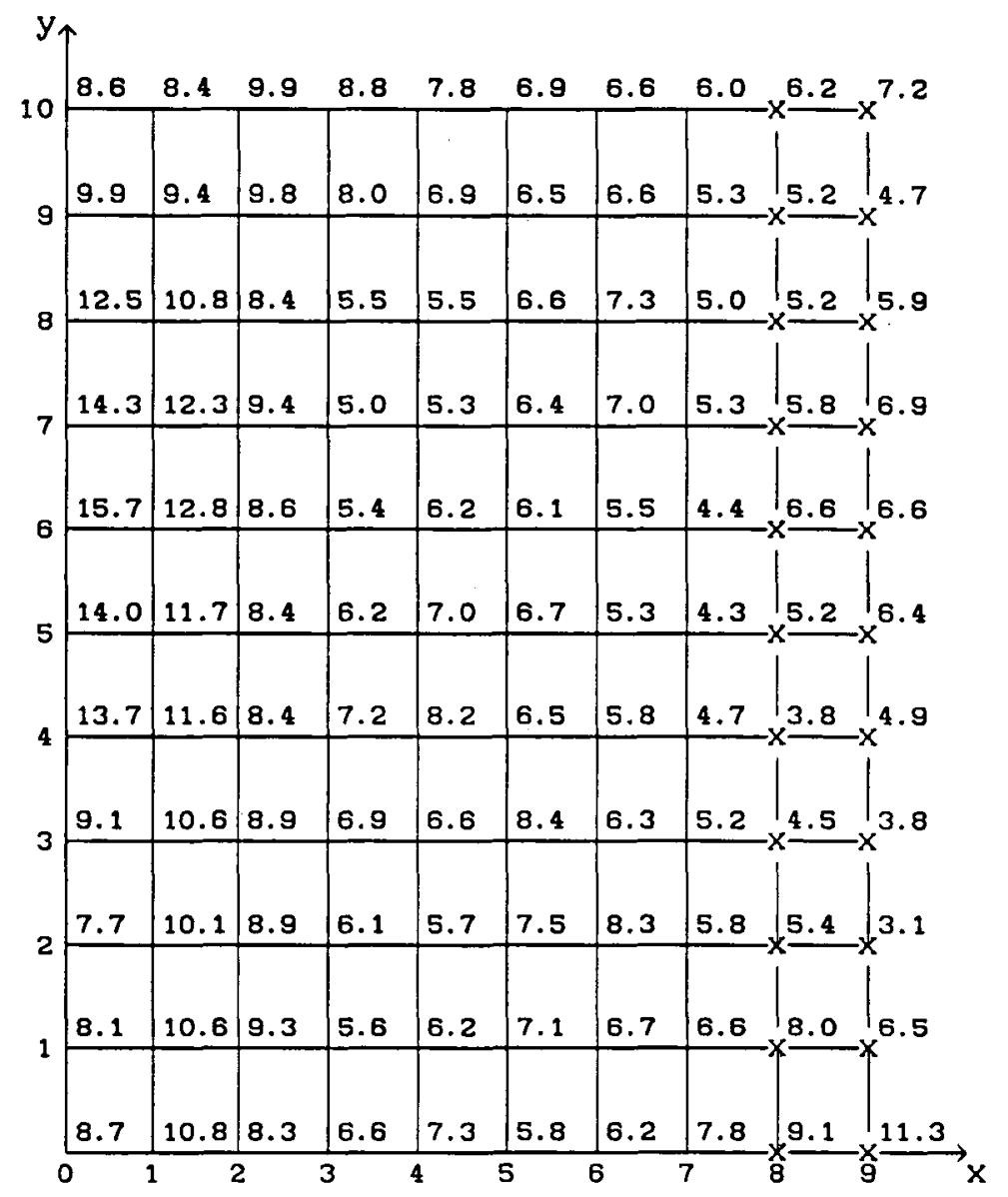




\begin{tabular}{|l|c|c|}
\cline { 2 - 3 } \multicolumn{1}{c|}{} & Solução $(\mu \mathrm{m})$ & $\begin{array}{c}\text { Tempo } \\
\text { Computacional (cs) }\end{array}$ \\
\hline Esp. Método Simplex & 8.93 & 71 \\
\hline Mínimos Quadrados & 9.76 & 38 \\
\hline
\end{tabular}

EXEMPLO 3:

De modo análogo, alteramos as medidas feitas no canto superior direito, considerando valores maiores, obtendo:

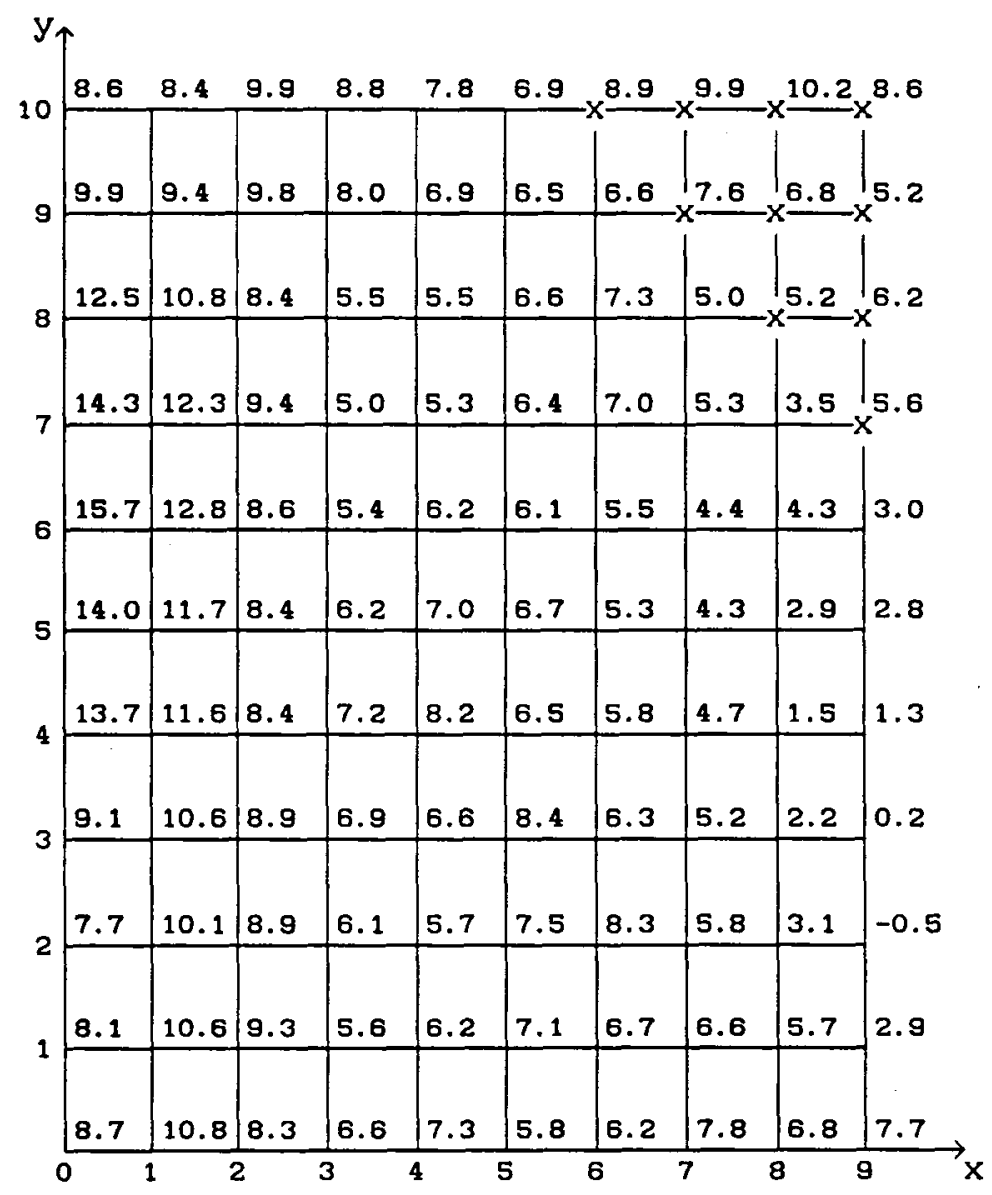




\begin{tabular}{|c|c|c|}
\cline { 2 - 3 } \multicolumn{1}{c|}{} & Solução $(\mu \mathrm{m})$ & $\begin{array}{c}\text { Tempo } \\
\text { Computacional (cs) }\end{array}$ \\
\hline Esp. Método Somplex & 8.55 & 99 \\
\hline Mínimos Quadrados & 9.07 & 38 \\
\hline
\end{tabular}

\section{EXEMPLO 4:}

Aqui, alteramos alguns dos valores das medições, para valores menores, como se a superfície tivesse uma depressão no meio:

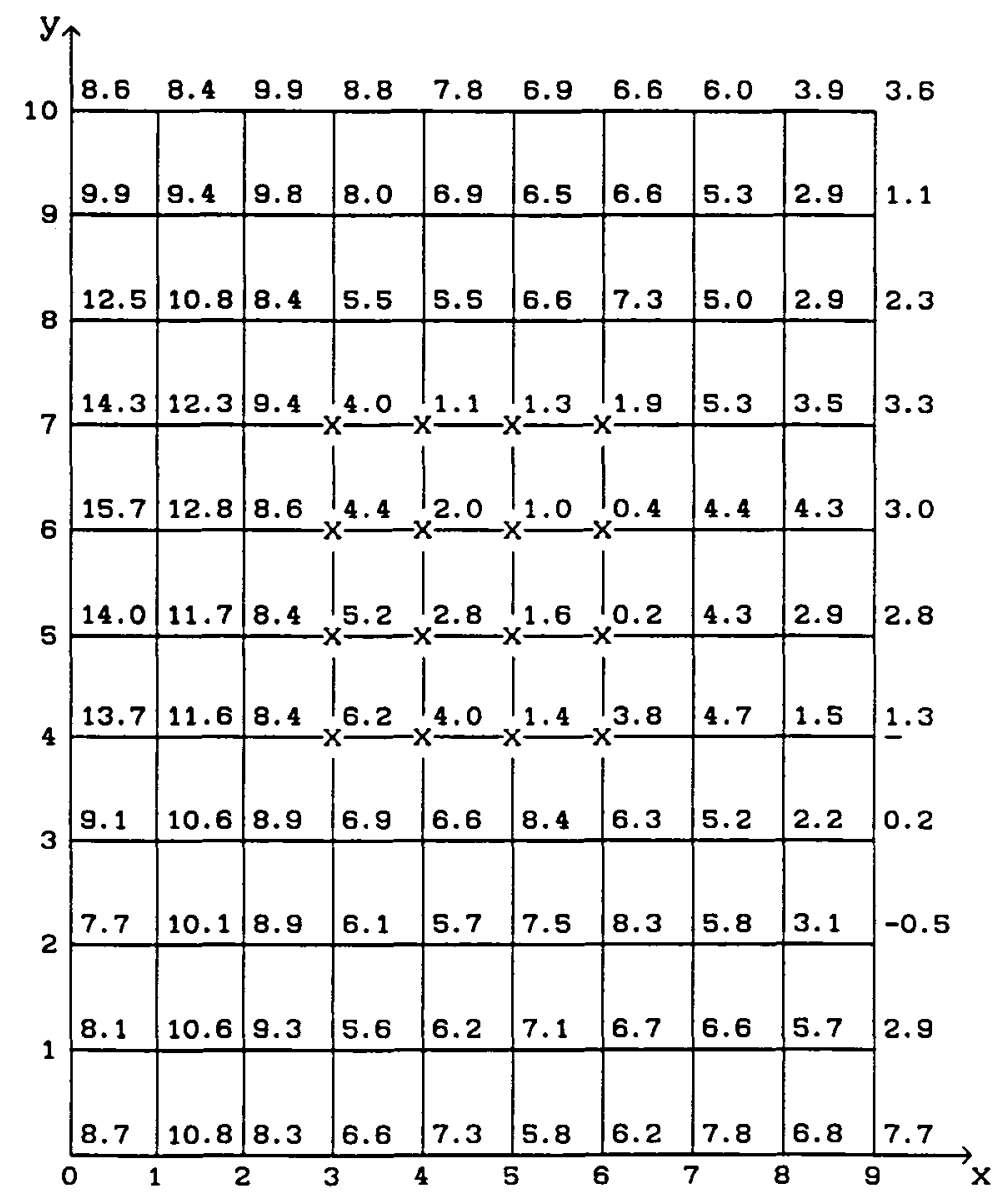




\begin{tabular}{|c|c|c|}
\cline { 2 - 3 } \multicolumn{1}{c|}{} & Solução $(\mu \mathrm{m})$ & $\begin{array}{c}\text { Tempo } \\
\text { Computacional (cs) }\end{array}$ \\
\hline Esp. Método Simplex & 9.59 & 98 \\
\hline Mínimos Quadrados & 10.95 & 39 \\
\hline
\end{tabular}

Os próximos exemplos foram extraídos de [ 4].

\section{EXEMPLO 5:}

As medições são dadas por:

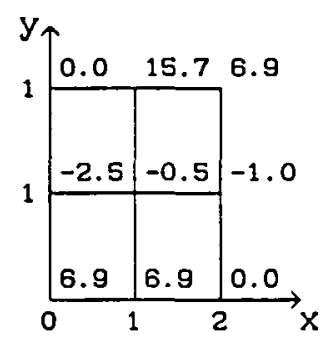

\begin{tabular}{|l|c|c|}
\cline { 2 - 3 } \multicolumn{1}{c|}{} & Solução $(\mu \mathrm{m})$ & $\begin{array}{c}\text { Tempo } \\
\text { Computacional (cs) }\end{array}$ \\
\hline Esp Método Simplex & 14.34 & 06 \\
\hline Mínimos Quadrados & 16.48 & 06 \\
\hline
\end{tabular}




\section{EXEMPLO 6:}

Neste exemplo, as medições são:

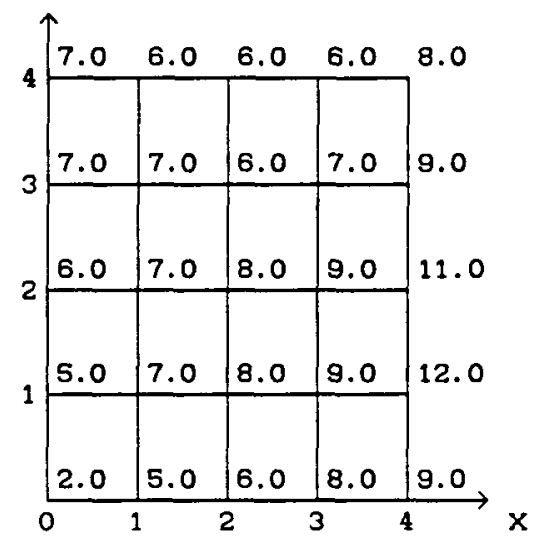

\begin{tabular}{|c|c|c|}
\cline { 2 - 3 } \multicolumn{1}{c|}{} & Solução $(\mu \mathrm{m})$ & $\begin{array}{c}\text { Tempo } \\
\text { Computacional (cs) }\end{array}$ \\
\hline Esp. Método Simplex & 4.86 & 16 \\
\hline Mínimos Quadrados & 5.90 & 06 \\
\hline
\end{tabular}




\section{EXEMPLO 7:}

Temos, aqui, as seguintes medições:

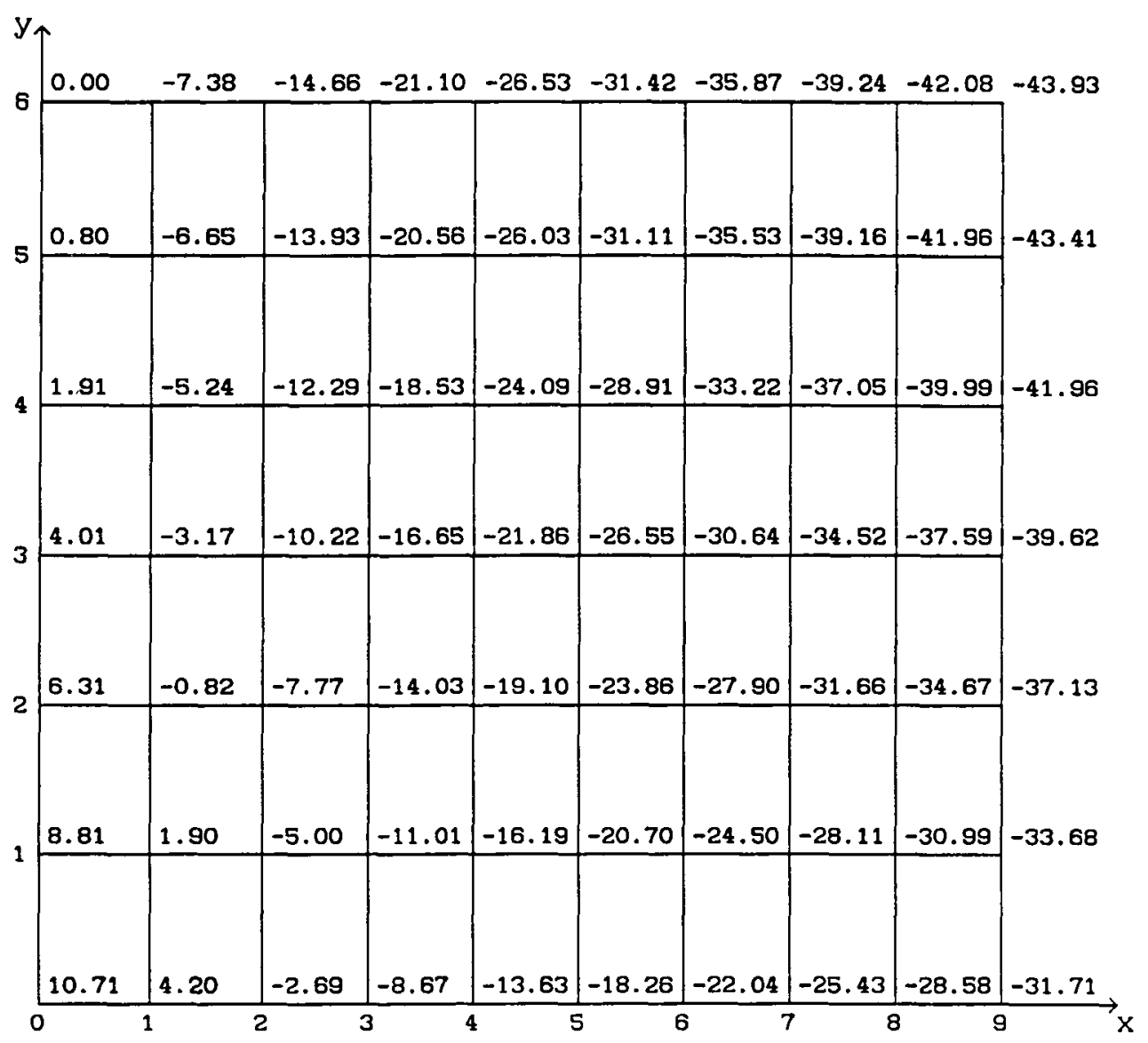

\begin{tabular}{|c|c|c|}
\cline { 2 - 3 } \multicolumn{1}{c|}{} & Solução $(\mu \mathrm{m})$ & $\begin{array}{c}\text { Tempo } \\
\text { Computacional (cs) }\end{array}$ \\
\hline Esp. Método Simplex & 8.75 & 55 \\
\hline Minimos Quadrados & 9.21 & 22 \\
\hline
\end{tabular}


Observamos que os resultados computacionais fornecidos em [ 4], do qual foram extraídos os exemplos 5,6 e 7 , coincidem com os resultados obtidos quando utilizamos a especialização do método Simplex, sendo que o referido artigo, faz uso de outro método.

Neste Capítulo, estudamos um método para resolver o problema de Planicidade, o qual, nos forneceu resultados computacionais satisfatórios, de acordo com as comparações feitas nos exemplos da seção 3.6. Ressaltamos que, as soluções fornecidas nos exemplos da seção 3.6 não são exatamente a Planicidade das superfícies pois, observando a fig 05, Capitulo 1, verificamos que a menor distancia entre os planos não é $z$. Portanto, a solução só coincidirá com a Planicidade, se os planos forem paralelos ao plano $x y$, caso contrário, devemos calcular a distancia entre os planos de controle, cujas equações são fornecidas pelo algoritmo da seção 3.5 , recorrendo a [10].

Quanto ao tempo computacional, podemos dizer que a diferença entre os dois métodos é satisfatória, levando em conta o fato dos métodos serem diferentes, a precisão do método Simplex e o tempo que se leva para obter as medições.

No próximo Capitulo, estudaremos um método para resolução de Sistemas Lineares Inconsistentes, o qual explora decomposicões da matriz e partições do sistema. 


\section{CAPITULO 4}

\section{UM METODO ASCENDENTE}

Neste capítulo, estudamos um outro método ascendente, para a resolução de (1.2), o qual faz uso de decomposições da matriz A e particções do sistema.

\subsection{Preliminares}

Introduzimos, aqui, alguns conceitos que serão usados no desenvolvimento do algoritmo, proposto na seção 4.6. Definimos H-Conjuntos e H-Conjuntos Minimais para sistemas lineares e caracterizamos as soluções ótimas.

\subsubsection{Definição de H-Conjuntos e H-Conjuntos Minimais para} Sistemas Lineares.

DEFINIÇÃo $1-U m$ conjunto $I=\left\{i_{1}, \ldots, i_{p+1}\right\} \subset\{1,2, \ldots, m\}$ é um H-Conjunto para $A \in \mathbb{R}^{\operatorname{mxn}}$, se existir um sinal padrão $\left\{s_{j}= \pm 1 ; j=1, \ldots, p+1\right\}$ tal que não exista $x \in \mathbb{R}^{n}$ que satisfaça a desigualdade

$$
s,\left(u_{1}^{t}, x\right)>0, \quad j=1, \ldots, p+1
$$


onde $u_{1}^{t}, \ldots, u_{m}^{t}$ são vetores linhas da matriz A.

O seguinte teorema é uma forma alternativa para a definição de H-Conjunto.

TEOREMA 1 : Sejam: I, conforme Definição 1 e $\mathbf{S} \in \mathbb{R}^{(p+1) \times n}$, uma matriz dada por $\left[u_{1}, \ldots, u_{i_{P+1}}\right]^{t}$. O conjunto I é um H-Conjunto para A se, e somente se, existe uma solução não trivial $\sigma \neq 0$ para,

$$
\sigma^{t} \mathbf{S}=0, \text { com } \sigma=\left\{\sigma_{1}, \ldots, \sigma_{p+1}\right\}
$$

o sinal padrão é, então, definido por $s_{j}=$ sinal $\sigma_{j}$, com $j=1, \ldots, p+1$.

Prova:

i) Suponhamos que $\sigma^{t} \mathbf{s}=0$, somente $\operatorname{com} \sigma=0, \mathbf{s}_{j}=\operatorname{sinal} \sigma_{j}$ e que não exista $x \in \mathbb{R}^{\mathrm{n}}$ que satisfaça a desigualdade (4.1).

Seja $D$ o casco convexo dos vetores $s_{j} u_{i}$, com $i_{j} \in I$. Tomemos $s_{j}= \pm 1$, qualquer. Portanto, o conjunto $\left\{s_{1} u_{i}, s_{2} u_{i}, \ldots, s_{p+1} u_{i p+1}\right\}$ é linearmente independente.

Como $0 \notin D$, usando o Teorema do Hiperplano Separador [13, capitulo1], temos que existe $\mathbf{x} \in \mathbb{R}^{\mathrm{n}}$ tal que:

$$
s_{j}\left(u_{1}^{t}, x\right)>0, i \in I \text {, }
$$

- que contraria a hipótese.

Portanto, os vetores são linearmente dependentes, ou seja, $\sigma S=0$, com $\sigma \neq 0$. 
ii) Por outro lado, suponhamos que:

$$
\sigma^{t} S=0, \quad \operatorname{com} \sigma \neq 0 \text { e } s_{j}=\operatorname{sinal}\left(\sigma_{j}\right)
$$

Logo,

$$
\sigma^{t} S x=0, \forall x \in \mathbb{R}^{n}
$$

ou, seja,

$$
\sum_{j=1}^{p+1} \sigma_{j} u_{1 j}^{t} x=0
$$

Multiplicando termo a termo por $\left(s_{j}\right)^{2}=1$, obtemos:

$$
\sum_{j=1}^{p+1}\left(\sigma_{j} s_{j}\right)\left(s_{j} u_{i j}^{t} x\right)=0
$$

de onde segue que

$$
\sigma_{j} s_{j} \geq 0, \text { com algum } \sigma_{k} s_{k}>0 \text {, }
$$

uma vez que $s_{j}=\operatorname{sinal}\left(\sigma_{j}\right)$ com $\sigma \neq 0$.

Logo não existe $x \in \mathbb{R}^{n}$ tal que $s_{j} \stackrel{u}{t}_{j}^{t} x>0,1 \leq j \leq p+1$, o que conclui a prova do Teorema. 
DEFINIÇÃo 2: Um H-Conjunto I é denominado H-Conjunto Minimal se não contém nenhum subconjunto próprio I' (ou seja, I'C I tal que I' $\neq$ I) que seja H-Conjunto.

\subsubsection{Propriedades de H-Conjuntos Minimais.}

PROPRIEDADE 1: Um conjunto I é um H-Conjunto Minimal para A, se, e somente se, posto $(S)=p$ e $\sigma_{1} \neq 0, \operatorname{com} i=1, \ldots, p+1$, em $(4.2)$.

Prova:

i) Suponhamos que posto(S) $=k<p, \sigma_{s}=0$ e que $I=\left\{i_{1}, \ldots, i_{p+1}\right\}$ seja um H-Conjunto Minimal para A.

Se posto(S) = k, então existem em $\mathbf{S}, \quad k$ linhas linearmente independentes, isto é, existe I' C I (por simplicidade de notação supomos que sejam os primeiros elementos de I) onde,

$$
I^{\prime}=\left\{i_{1}, \ldots, i_{k+1}\right\}, \sigma^{t} S=0, \text { com } \sigma \neq 0 \text {. }
$$

Portanto, existe I'C I tal que I'é um H-Conjunto Minimal para A, O que é um absurdo, pois I é um H-Conjunto Minimal para A.

Logo posto $(\mathbf{S})=p$.

Se $\sigma_{s}=0$, temos que $i_{s}$ pode ser retirado do conjunto I determinando um subconjunto que também é H-Conjunto, o que nos leva a um absurdo, pois I é um H-Conjunto Minimal.

Portanto, $\sigma_{s} \neq 0$ 
ii) Reciprocamente, suponhamos que $I=\left\{i_{1}, \ldots, i_{p+1}\right\}$ não seja um H-Conjunto Minimal para $A$, mas que posto(S) $=p$ e $\sigma_{1} \neq 0$, com $1 \leq i \leq p+1$.

Então, existem $I^{\prime}=\left\{i_{1}, \ldots, \dot{i}_{k+1}\right\} \subset I, k<p$ e uma submatriz $S^{\prime}$ de $S$, formada pelas linhas indexadas em I'. Temos,

$$
\sigma^{\prime t} S^{\prime}=0, \operatorname{com} \sigma^{\prime} \neq 0
$$

Concluimos, então, que as linhas da matriz $\mathbf{S}$ são linearmente dependentes e, portanto, posto $(S)<k+1$, ou seja, posto( $S) \leq k<p$, - que contraria a hipotese de que posto(S) $=\mathrm{p}$.

Portanto, I é um H-Conjunto Minimal para A.

PROPRIEDADE 2: Todo sub-conjunto de $\{1, \ldots, m\}$ com mais que $(n+1)$ elementos é um H-Conjunto para A. Então, um H-Conjunto Minimal para A tem no máximo $(n+1)$ elementos.

\section{Prova:}

Sabemos que $u_{i}^{t} \in \mathbb{R}^{n}$, ou seja, o espaço linha da matriz A tem no máximo $\mathrm{n}$ vetores linearmente independentes.

Portanto, $\mathrm{n}+1$ linhas da matriz A (matriz S) são linearmente dependentes; então,

$$
\exists \sigma \neq 0 / \sigma^{t} \mathbf{S}=0
$$

Suponhamos, agora, cardinalidade de I maior que $n+1$. Então existe um subconjunto I' com cardinalidade $n+1$, o qual é linearmente dependente, isto é, é um $\mathrm{H}$-Conjunto também. 
PROPRIEDADE 3: $I=\left\{i_{1}\right\}$ é um $\mathrm{H}$-Conjunto Minimal para $A$, se e somente se, $u_{1}=0$.

Prova:

Basta observarmos que:

$$
\sigma_{1} u_{1}^{t}=0, \operatorname{com} \sigma_{1} \neq 0
$$

se e somente se,

$$
u_{1}^{t}=0
$$

PROPRIEDADE 4: Se I é um H-Conjunto Minimal para A, então toda submatriz (pxn) de $S$ tem posto $p$.

Prova:

Suponhamos que exista uma submatriz $S^{\prime} \in \mathbb{R}^{\text {pxn }}$ de $S \in \mathbb{R}^{(p+1) \times n}$, tal que posto $\left(\mathbf{S}^{\prime}\right)=r$, onde $r<p$. Sem perda de generalidade, podemos tomar os $r$ primeiros elementos de $I$.

Então, os vetores $\left\{u_{i}, \ldots, u_{1}\right\}$ são linearmente independentes $e$ $\left\{u_{i}, \ldots, u_{1}, \ldots, u_{i}\right\}$ são linearmente dependentes.

Assim, temos $\sigma^{t} \mathbf{S}=0$.

Porém, podemos tomar $\sigma_{p+1}=0 \mathrm{e}$, consequentemente, I não é um H-Conjunto Minimal para A, o que é um absurdo.

Portanto, cada submatriz ( $p \times n)$ de $S$ tem posto $p$. 


\subsubsection{Caracterização das soluções ótimas usando H-Conjuntos} Minimais.

Usando a definição de H-Conjuntos Minimais obtemos uma caracterização das soluções ótimas, através do seguinte teorema:

TEOREMA 2 - TEOREMA DA CARACTERIZAÇÃo: O vetor $\hat{x} \in \mathbb{R}^{n}$ é uma solução de Chebyshev para o sistema $A x=b$ se, e somente se, o conjunto de indices extremos

$$
E(\hat{\mathbf{x}})=\left\{i \in[1, \mathrm{~m}] ;\left|r_{i}(\hat{\mathbf{x}})\right|=\|\mathbf{r}(\hat{x}) \cdot\|\right\}
$$

contém um H-Conjunto Minimal

com sinais

$$
I=\left\{i_{1}, \ldots, i_{p+1}\right\}
$$

$$
\mathbf{s}_{j}=\operatorname{sinal} \mathrm{r}_{\mathrm{i}}(\hat{\mathbf{x}}), \operatorname{com} 1 \leq \mathrm{j} \leq \mathrm{p}+1
$$

Prova:

i) Suponhamos que $\hat{x}$ não seja uma solução de Chebyshev para o sistema $A \mathbf{x}=\mathbf{b}$, mas que exista $\circ$ conjunto de índices extremos $E(\hat{\mathbf{x}})$ contendo um H-Conjunto Minimal I, com os sinais especificados no teorema.

Como $\hat{x}$ não é uma solução, então existe $d \in \mathbb{R}^{n}$ tal que:

$$
\|\mathbf{r}(\hat{\mathbf{x}}+d)\|<\|\mathbf{r}(\hat{\mathbf{x}})\|=\left|\mathrm{r}_{i_{j}}(\hat{x})\right|, \forall 1_{j} \in I \subset E(\hat{x}) ;
$$

em particular, temos:

$$
\left|r_{1_{j}}(\hat{x}+d)\right|<\left|r_{i j}(\hat{x})\right| \text {, para todo } 1_{j} \in I
$$


Sabemos que:

$$
r(\hat{x}+d)=b-A(\hat{x}+d)=r(\hat{x})-A d,
$$

de onde segue que

$$
r_{1}(\hat{x}+d)=r_{i j}(\hat{x})-u_{i j}^{t} d, \text { com } 1 \leq j \leq p+1
$$

De (4.3) e (4.4) temos:

$$
\left|r_{1 j}(\hat{x})-u_{1,}^{t} d\right|<\left|r_{1 j}(\hat{x})\right|
$$

Então,

$$
s_{j} u_{i, j}^{t} d>0 \text {, para todo } i_{j} \in I \text {. }
$$

Teremos um absurdo por escrever as condições do teorema,

$$
\sum_{j=1}^{p+1} \sigma_{j} u_{1 j}^{t}=0
$$

Multiplicando (4.6) termo a termo por $\left(s_{j}\right)^{2}$ e d, obtemos:

$$
\sum_{j=1}^{p+1} \sigma_{j} s_{j} s_{j} u_{i j}^{t} d=0
$$

- que é um absurdo pois de (4.5) temos que $\mathrm{s}_{j} \mathbf{u}_{1}^{t} \mathrm{~d}>0,1_{j} \in I$ e sabemos quer, $s_{j}>0$.

Logo, 


$$
\sum_{j=1}^{p+1} \sigma_{j} s_{j} s_{j} u_{i j}^{t} d>0
$$

e, consequentemente, $\hat{\mathbf{x}}$ é uma solução de Chebyshev para o sistema.

ii) Seja $\dot{x}$ a solução de Chebyshev para o sistema $A \quad x=b$ e suponhamos que não exista um H-Conjunto Minimal com os sinais especificados.

Então, $0 \notin D$, onde $D$ é o casco convexo de vetores $s, u_{1}^{t}, 1 \in E(\hat{x})$

Pelo Teorema do Hiperplano Separador [13, capitulo 1$]$, existe $d \in \mathbb{R}^{n}$ tal que:

$$
s_{j} u_{1}^{t} d>0, \quad 1 \in E(\hat{x}) .
$$

Logo, vem:

$i i_{1}$ ) Para $1 \in E(\hat{x})$, temos:

$$
\begin{aligned}
\|r(\hat{x}+\tau d)\| & =\left|r_{1}(\hat{x}+\tau d)\right|= \\
& =\left|b_{1}-u_{1}^{t}(\hat{x}+\tau d)\right|= \\
& =\left|b_{1}-u_{1}^{t} \hat{x}-\tau u_{1}^{t} d\right|= \\
& =\left|r_{1}(\hat{x})-\tau u_{1}^{t} d\right|= \\
& =\left|s_{j}\|r(\hat{x})\|-\tau u_{1}^{t} d\right|= \\
& =\left|\|r(\hat{x})\|-\tau s_{j} u_{1}^{t} d\right|<\|r(\hat{x})\|, \\
\text { se, } 0<\tau<\frac{2\|r(\hat{x})\|}{\max \left(s_{j} u_{1}^{t} d\right)} &
\end{aligned}
$$


pois,

$$
\left|\|r(\hat{x})\|-\tau s, u_{1}^{t} d\right|<\|r(\hat{x})\|
$$

Então, vem:

$$
-\|r(\hat{x})\|<\|r(\hat{x})\|-\tau s_{j} u_{1}^{t} d<\|r(\hat{x})\|,
$$

isto é,

$$
-2\|r(\hat{x})\|<-\tau s, u_{1}^{t} d<0,
$$

ou seja,

$$
0<\tau s, u_{i}^{t} d<2\|r(\hat{x})\|
$$

ou, ainda,

$$
0<\tau<\frac{2\|r(\hat{x})\|}{s_{j} u_{1}^{t} d} .
$$

Portanto,

$$
0<\tau<\frac{2\|r(\hat{x})\|}{\max \left(s, u_{1}^{t} d\right)} .
$$

i $_{2}$ ) Para $i \notin E(\hat{x}), \tau>0$. 


$$
\begin{aligned}
& \|\mathbf{r}(\hat{x}+\tau d)\|=\left|r_{i}(\hat{x}+\tau d)\right|= \\
& =\left|r_{i}(\hat{x})-\tau u_{i}^{t} d\right| \leq \\
& \leq\left|r_{1}(\hat{x})\right|+\left|\tau u_{1}^{t} d\right| \leq \\
& \leq \max \left|r_{1}(\hat{x})\right|+\tau \max \left|u_{1}^{t} d\right|<\|r(\hat{x})\| \text {. } \\
& 1 \notin E(\hat{x}) \quad 1 \notin E(\hat{x})
\end{aligned}
$$

se $0<\tau<\frac{\left(\|r(\hat{x})\|-\max _{\left.1 \notin E(\hat{x})^{1}(\hat{x}) \mid\right)}\right.}{\max _{1 \notin E(\hat{x})^{t} d \mid} \text {, }}$

uma vez que

$$
\underset{1 \notin E(\hat{x})}{\max \left|r_{1}(\hat{x})\right|+\tau \max \left|u_{1}^{t} d\right|<\|r(\hat{x})\| .}
$$

Logo, temos:

$$
\underset{1 \notin E(\hat{x})}{\tau \max \left|u_{1}^{t} d\right|<\|r(\hat{x})\|-} \max \left|r_{1}(\hat{x})\right|,
$$

de onde segue que

$$
0<\tau<\frac{\left(\|r(\hat{x})\|-\max _{\left.\left.1 \notin E(\hat{x})_{1} \mid \hat{x}\right) \mid\right)}\right.}{\max _{1 \notin E(\hat{x})_{1}^{t} d \mid} \mid}
$$

Então, se $\tau>0$ é suficientemente pequeno, o fato de $\hat{x}$ ser uma solução de Chebyshev para $\mathbf{A} \mathbf{x}=\mathbf{b}$ é um absurdo, pois sempre temos disponível uma direção d de descida. 
Portanto, existe um H-Conjunto Minimal com os sinais já especificados.

De i) e ii), temos a prova do teorema.

Portanto caracterizamos uma solução de Chebyshev por

$$
b_{i j}-u_{i j}^{t} \hat{x}=s_{j} \alpha, \quad 1 \leq j \leq p+1
$$

onde $\alpha$ é definido em (1.2) e $s_{j}=$ sinal $\sigma_{j}$, com $\sigma$ resolvendo (4.2).

Observando que dimensão do Ker de $s^{t}$ é igual a 1 , considerando a Propriedade 1 , normalizamos $\sigma^{t} \in \operatorname{ker} s^{t}$ por $\sum_{j}\left|\sigma_{j}\right|=1$.

Então de (4.2), vem:

$$
\sigma^{t} s=0,\|\sigma\|_{1}=1 \text { e } s_{j}=\operatorname{sinal} \sigma_{j}
$$

e de (4.7), obtemos:

$$
b_{1,}-u_{1, j}^{t} x=s, \alpha, 1 \leq j \leq p+1
$$

Logo,

$$
\sum_{j=1}^{p+1} \sigma_{j} b_{1, j}-\sum_{j=1}^{p+1} \sigma_{j} u_{1 j}^{t} x=\sum_{j=1}^{p+1} \sigma_{j} s, \alpha, \quad 1 \leq j \leq p+1
$$

- que acarreta 


$$
\alpha=\sum_{j=1} \sigma_{j} b_{i j},
$$

onde o sinal arbitrário de $\sigma$ é fixado pela condição $\alpha \geq 0$.

Pela Propriedade 1 , o sistema (4.7) impõe $p$ condições independentes sobre $x$, tal que a solução de Chebyshev não é necessariamente única no caso de $\mathrm{p}<\mathrm{n}$.

Todas as soluções partilham a seguinte propriedade:

PROPRIEDADE 5: Toda solução de Chebyshev tem o mesmo residuo $s, \alpha$, $(1 \leq j \leq p+1)$, para as equações correspondentes ao H-Conjunto Minimal I.

Nas próximas seções faremos a descrição dos passos que serão usados no algoritmo da seção 4.6 .

\subsection{Decomposição Canônica da Matriz.}

Nesta seção, estudamos a decomposição de uma matriz de d linhas e e colunas, que será utilizada no algoritmo da seção 4.6. Essa decomposição foi proposta por Noble para o calculo da inversa generalizada [ 5].

Seja uma matriz $D \in \mathbb{R}^{\mathrm{dxe}}$, com posto $r$. Ordenando as linhas $\mathrm{e}$ colunas de $\mathbf{D}$, podemos determinar uma submatriz principal $E \in \mathbb{R}^{\Gamma \times \Gamma}$, não singular. Portanto, as primeiras $r$ colunas de $D$ formam uma base do espaço coluna $e$ as $(e-r)$ colunas restantes podem ser expressas como combinação linear destes vetores básicos, ou seja, D pode ser escrita na forma: 


$$
D=\left(\begin{array}{l:l}
E & c \\
X &
\end{array}\right] \text {, }
$$

onde $\quad C=\left[c^{1}, c^{2}, \ldots, c^{e-r}\right] \in \mathbb{R}^{d x(e-r)}$

Então,

$$
c^{j}=\left[\begin{array}{l}
E \\
x
\end{array}\right] f^{j}
$$

onde $f^{\prime}$ são as coordenadas da coluna $c^{\prime}$ na base $\left[\begin{array}{l}E \\ x\end{array}\right], 1 \leq j \leq e-r$, isto é,

$$
C=\left[\begin{array}{l}
E \\
X
\end{array}\right] F
$$

onde $F=\left[f^{1}, f^{2}, \ldots, f^{e-r}\right] \in \mathbb{R}^{r \times(e-r)}$

Logo podemos escrever:

$$
D=\left[\begin{array}{ll}
E & E F \\
X & X F
\end{array}\right]
$$


Usando o mesmo procedimento para as $r$ primeiras linhas, teremos :

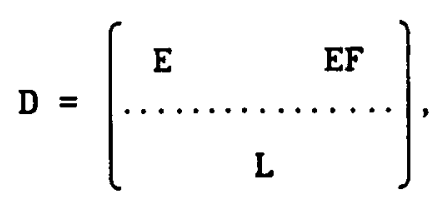

onde $L=\left[1^{1}, 1^{2}, \ldots, 1^{d-r}\right]^{t} \in \mathbb{R}^{(d-r) \times(e)}$.

Portanto,

$$
1^{1}=g^{1^{t}}\left[\begin{array}{ll}
E & E F
\end{array}\right.
$$

onde $g^{1}$ são as coordenadas da linha $1^{1}$ na base $[E$ EF], $1 \leq i \leq d-r$ ou seja,

$$
L=G\left[\begin{array}{ll}
E & E F
\end{array}\right]
$$

onde $G=\left[g^{1}, g^{2}, \ldots, g^{d-r}\right]^{t} \in \mathbb{R}^{(d-r) x r}$

Assim D também pode ser escrita na forma:

$$
D=\left[\begin{array}{cc}
E & E F \\
G E & G E F
\end{array}\right] \text {, }
$$


ou ainda,

$$
D=\left[\begin{array}{c}
I_{r} \\
G
\end{array}\right] \quad E\left[\begin{array}{ll}
I_{r} & F
\end{array}\right]
$$

As matrizes $E, \quad F$ e $G$ contêm número de parâmetros independentes, caracterizando uma matriz (dxe), de posto $r$, isto é :

$$
r^{2}+r(e-r)+(d-r) r=r(d+e-r)
$$

Se $r=\min \{d, e\}$, então (4.10) resulta em (d.e), de onde concluímos que todas as linhas de $D$ são independentes.

Para $r=1$, (4.9) decompõe-se no produto de um vetor coluna por um vetor linha e o número de parâmetros independentes torna-se $(d+e-1)$.

Decompondo $D$ no produto de uma matriz triangular inferior por uma outra matriz, podemos reescrevê-la da seguinte forma:

$$
\left[\begin{array}{ll}
E^{-1} & 0_{r, d-r} \\
0_{d-r, r} & I_{d-r}
\end{array}\right]\left[\begin{array}{ll}
E & E F \\
G E & G E F
\end{array}\right]=\left[\begin{array}{cc}
I_{r} & F \\
G E & G E F
\end{array}\right]
$$

ou seja,

$$
\left[\begin{array}{cc}
I_{r} & 0_{r, d-r} \\
-G E & I_{d-r, d-r}
\end{array}\right]\left[\begin{array}{cc}
E^{-1} & 0_{r, d-r} \\
0_{d-r, r} & I_{d-r}
\end{array}\right]\left[\begin{array}{cc}
E & E F \\
G E & G E F
\end{array}\right]=\left[\begin{array}{cc}
I_{r} & F \\
0 & 0
\end{array}\right]
$$


Efetuando o produto entre as duas primeiras matrizes, obtemos a decomposição:

$$
\left[\begin{array}{cc}
E^{-1} & 0_{r, d-r} \\
-G & I_{d-r}
\end{array}\right]\left[\begin{array}{ll}
E & E F \\
G E & G E F
\end{array}\right]=\left[\begin{array}{ll}
I_{r} & F \\
0_{d-r, r} & 0_{d-r, e-r}
\end{array}\right]
$$

Trabalhando $\operatorname{com} D^{t}$, substituímos $E, F$ e $G$, respectivamente, por $E^{t}, F^{t}$ e $G^{t}$, produzindo a decomposição :

$$
D\left[\begin{array}{ll}
E^{-1} & -F \\
0_{e-r, r} & I_{e-r}
\end{array}\right]=\left[\begin{array}{ll}
I_{r} & 0_{r, e-r} \\
G & 0_{d-r, e-r}
\end{array}\right]
$$

Essas matrizes fornecem as informações necessárias para a próxima seção. Por exemplo, (4.11) e (4.12) mostram que as colunas $\left[\begin{array}{ll}-G & I_{d-r}\end{array}\right]^{t}$ e $\left[\begin{array}{lll}-F^{t} & I_{e-r}\end{array}\right]^{t}$ são vetores básicos de $\operatorname{ker} D^{t}$ e ker $D$, respectivamente.

\subsection{Partição do Sistema Relativo a um H-Conjunto Minimal.}

Nesta seção, estudamos como particionar o sistema relacionado a um H-Conjunto Minimal e calcular a solução de Chebyshev para o sistema já particionado.

Sejam $I=\left\langle i_{1}, \ldots, i_{p+1}\right\}$ um H-Conjunto Minimal para a matriz $A$ 
e $\mathbf{S}=\left[\mathbf{u}_{1}, \ldots, \mathbf{u}_{1}\right]^{t}$ a matriz associada, de $(p+1)$ linhas por $n$ colunas, tal que posto(S) $=p$.

Aplicando a decomposição descrita na seção 4.2 e permutando de maneira conveniente as colunas, podemos escrever $\mathbf{S}$ como:

$$
S=\left[\begin{array}{ll}
Q & R
\end{array}\right], \text { com: } R=Q U, Q \in \mathbb{R}^{(p+1) \times p} \text { e } U \in \mathbb{R}^{p \times(n-p)}
$$

onde as colunas de $U$ representam os vetores coordenadas das últimas colunas de $\mathbf{S}$, em termos da base formada pelas primeiras p colunas.

Ordenando as linhas de A convenientemente, suponhamos que as primeiras $t$ linhas de A pertençam ao espaço linha de $S$. Então, reescrevemos A da seguinte maneira:

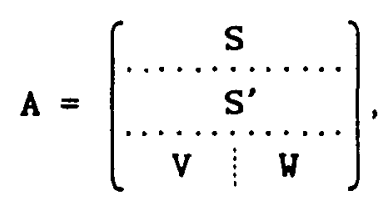

onde,

$$
\left.\mathbf{S}^{\prime}=\mathbf{r}_{\mathrm{p}+2}, \cdots, \mathbf{u}_{\mathrm{t}}\right]^{\mathrm{t}},
$$

$\operatorname{com} u_{1}=\sum_{j=1}^{p+1} \delta_{j}^{1} s_{j}, p+2 \leq i \leq t, e s_{j}$ denota a j-ésima linha da matriz S, ou seja,

$$
u_{1}=\Delta_{1}^{t} s
$$

onde $\Delta_{1}^{t}=\left[\delta_{1}^{1}, \ldots, \delta_{p+1}^{1}\right]$ 
Assim,

$$
S^{\prime}=\Delta S,
$$

onde $\Delta=\left[\begin{array}{c}\Delta_{p+2}^{t} \\ \vdots \\ \Delta_{t}^{t}\end{array}\right]$.

Como $\mathbf{S}=\left[\begin{array}{ll}Q & Q U\end{array}\right], S^{\prime}$ pode ser escrita na forma:

$$
\mathbf{S}^{\prime}=\left[\begin{array}{ll}
\Delta \mathrm{Q} & \Delta \mathrm{QU}
\end{array}\right]
$$

Portanto,

$$
\left[\begin{array}{c}
\mathbf{s} \\
\cdots \\
\mathrm{S}^{\prime}
\end{array}\right]=\left[\begin{array}{cc}
\mathrm{Q} & \mathrm{QU} \\
\ldots \ldots & \ldots \\
\Delta \mathrm{Q} & \Delta \mathrm{QU}
\end{array}\right] .
$$

\section{Chamando}

$$
\left[\begin{array}{c}
Q \\
\Delta Q
\end{array}\right]=T
$$

temos:

$$
\left[\begin{array}{c}
\mathbf{S} \\
\cdots \\
\mathbf{S}^{\prime}
\end{array}\right]=\left[\begin{array}{ll}
\mathrm{T} & \mathrm{TU}
\end{array}\right]
$$


onde, $T \in \mathbb{R}^{\text {txp }}$ e a matriz $\left\lfloor T\right.$ TU $\int$ reúne as linhas de $A$ que estão no espaço linha de $\mathbf{S}$.

Deste modo, podemos escrever:

$$
A=\left[\begin{array}{cc}
T & T U \\
V & W
\end{array}\right]
$$

onde: $V \in \mathbb{R}^{(m-t) \times p}$ e $W \in \mathbb{R}^{(m-t) \times(n-p)}$.

Podemos decompor a matriz A da seguinte maneira:

$$
\begin{aligned}
& \left.\qquad \begin{array}{cc}
T & T U \\
V & W
\end{array}\right]\left[\begin{array}{cc}
I_{p} & -U \\
O_{n-p, p} & I_{n-p}
\end{array}\right]=\left[\begin{array}{cc}
T & 0_{t, n-p} \\
V & W-V U
\end{array}\right] \text {, } \\
& \text { onde } K=\left[\begin{array}{ll}
I_{p} & -U \\
0_{n-p, p} & I_{n-p}
\end{array}\right] \text {. }
\end{aligned}
$$

Seja o espaço gerado pela matriz $\mathbf{S}$ dado por:

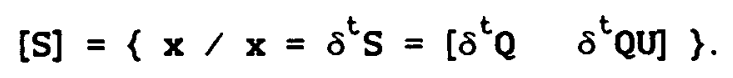

Suponhamos que a matriz W - VU tenha uma linha nula, dada por:

$$
w_{i}^{t}-v_{i}^{t} u=0,
$$

então, temos:

$$
w_{1}^{t}=v_{1}^{t} u
$$


e, portanto,

$$
\left(v_{1}^{t}, w_{1}^{t}\right)=\left(\delta^{t} Q \quad \delta^{t} Q U\right) \in[S]
$$

- que é um absurdo. Logo, W - vU não tem linhas nulas.

Temos, então, o seguinte sistema transformado para $A x=b$ :

$$
A K^{-1} \mathbf{x} \approx \mathbf{b} \text {. }
$$

$$
\text { Chamando } \mathrm{K}^{-1} \mathrm{x}=\left[\begin{array}{c}
\mathrm{y} \\
\cdots \\
z
\end{array}\right] \text {, onde } \mathrm{y} \in \mathbb{R}^{p} \text { e } z \in \mathbb{R}^{\mathrm{n}-\mathrm{p}} \text {, temos: }
$$

$$
\left[\begin{array}{cc}
T & 0_{t, n-p} \\
V & W-v U
\end{array}\right]\left[\begin{array}{l}
y \\
z
\end{array}\right] \approx\left(\begin{array}{l}
c \\
d
\end{array}\right],
$$

onde $c \in \mathbb{R}^{t}$ e $d \in \mathbb{R}^{m-t}$.

Computamos a solução de Chebyshev para o sistema $T y \approx c$, o qual tem posto coluna completo $\mathrm{p}$ e o menor número de variáveis em relação ao H-Conjunto Minimal dado.

Aplicamos recursivamente o mesmo procedimento para reduzir o sistema:

$$
(w-v) z \approx d-\hat{v y} \text {, }
$$

fazendo $\bar{A}=W-V U \in \mathbb{R}^{\bar{m} \times \bar{n}}$ e $\bar{b}=d-\hat{V y} \in \mathbb{R}^{\bar{m}}$, temos:

$$
\overline{\mathbf{A}} \mathbf{z}=\overline{\mathbf{b}},
$$

$\operatorname{com} \bar{m}=m-t e \bar{n}=n-p$. 
Portanto, a solução para o sistema original é:

$$
x=K\left[\begin{array}{c}
\hat{y} \\
z
\end{array}\right]=\left[\begin{array}{cc}
I_{p} & -U \\
0_{n-p, p} & I_{n-p}
\end{array}\right]\left[\begin{array}{l}
\hat{y} \\
z
\end{array}\right]=\left[\begin{array}{c}
\hat{y}-U z \\
z
\end{array}\right]
$$

isto é,

$$
x=\left[\begin{array}{l}
\hat{y} \\
0_{n-p}
\end{array}\right]+\left[\begin{array}{c}
-U \\
I_{n-p}
\end{array}\right] z
$$

A partição da matriz aqui apresentada depende da escolha do H-Conjunto Minimal. Um procedimento para a escolha deste será apresentada na seção 4.5.

\subsection{Troca entre H-Conjuntos Minimais.}

O algoritmo que desenvolveremos na seção 4.6, tem como objetivo determinar o ótimo do problema (1.2), o que corresponde ao desvio máximo entre todos os $\mathrm{H}$-Conjuntos Minimais. Verificaremos que 0 algoritmo aumenta o desvio a cada passo. Assim, serão importantes as trocas entre H-Conjuntos Minimais relativos a sistemas sucessivos, as quais serão descritas nesta seção.

Consideremos, para a matriz A, um H-Conjunto Minimal $I=\left\{i_{1}, \ldots, i_{p+1}\right\}$. Essa matriz pode ser decomposta como (4.13); além disso ela satisfaz (4.2). Em I, temos que o desvio é dado por (4.8) e que $1 \leq i_{k} \leq t$, onde $1 \leq k \leq p+1$. Tomemos, agora, para a matriz $\bar{A}=W-v U$, um H-Conjunto Minimal $\bar{I}=\left\{\bar{i}_{1}, \ldots, \bar{i}_{\bar{p}_{+1}}\right\}$, cujos indices correspondem às linhas de $\bar{A}$. Portanto, temos que $1 \leq \bar{i}_{k} \leq \bar{m}$, onde 
$1 \leq k \leq \bar{p}+1$, e os indices correspondentes para linhas de A são:

$$
j_{k}=t+\bar{i}_{k}, \text { com } 1 \leq k \leq \bar{p}+1 \text {. }
$$

Podemos escrever a matriz $\overline{\mathrm{S}} \in \mathbb{R}^{(\bar{p}+1) \times \bar{n}}$ associada a $\overline{\mathrm{A}}$, como:

$$
\overline{\mathbf{S}}=\overline{\mathbf{R}}-\overline{\mathrm{Q}} \mathbf{U},
$$

onde, $\overline{\mathbf{Q}} \in \mathbb{R}^{(\bar{p}+1) \times p}$ e $\overline{\mathbf{R}} \in \mathbb{R}^{(\bar{p}+1) \times \bar{n}}$ são submatrizes de $V$ e $W$ dadas em (4.14), respectivamente.

o desvio $\bar{\alpha}$ em $\bar{I}$ é dado por:

$$
\bar{\alpha}=\sum_{k=1}^{\bar{p}+1} \bar{\sigma}_{k} \bar{b}_{i_{k}} \geq 0
$$

$\operatorname{com} \vec{b}=d-\hat{y}$.

Esse desvio é tal que $\bar{\sigma}^{t}=\left[\bar{\sigma}_{1}, \ldots, \bar{\sigma}_{\bar{p}+1}\right]$ satisfaz $\bar{\sigma}^{t} \overline{\mathbf{s}}=0$, com a condição de normal ização $\sum_{\mathbf{k}}\left|\bar{\sigma}_{\mathbf{k}}\right|=1$.

Podemos combinar as $p+\bar{p}+2$ linhas da matriz $A$, as quais são relacionadas a $I$ e $\bar{I}$, obtendo:

$$
P=\left[\begin{array}{cc}
Q & R \\
\bar{Q} & \bar{R}
\end{array}\right]
$$

a qual é equivalente a: 


$$
\begin{aligned}
& {\left[\begin{array}{cc}
Q & R \\
\bar{Q} & \bar{R}
\end{array}\right]\left[\begin{array}{cc}
I_{p} & -U \\
O_{n-p, p} & I_{n-p}
\end{array}\right]=\left[\begin{array}{cc}
Q & 0_{p+1, \bar{n}} \\
\bar{Q} & \bar{s}
\end{array}\right]} \\
& \text { onde } K=\left[\begin{array}{ll}
I_{p} & -U \\
0_{n-p, p} & I_{n-p}
\end{array}\right] \text { é a matriz definida na seção 4.3. }
\end{aligned}
$$

Considerando que posto(S) $=\mathrm{p}$, que em $\overline{\mathbf{Q}}$ e $\overline{\mathbf{R}}$ estão as linhas de $A$, as quais não pertencem ao espaço linha de $\mathbf{S}$, e que $P^{t}$ tem nulidade 2. concluímos que $\operatorname{posto}(P)=p+\bar{p}$.

Definimos uma base para $\operatorname{ker}\left(P^{t}\right)$ da seguinte maneira:

i) Tomamos o vetor $\left[\sigma^{t}, 0 \frac{t}{p+1}\right]^{t} \circ$ qual satizfaz $\left[\sigma^{t}, 0 \frac{t}{p+1}\right] P=0$;

ii) Denotamos o outro vetor da base por $\left[\tau^{t}, \tau^{t}\right]^{t}$, com $\tau \in \mathbb{R}^{p+1} e$ $\bar{\tau} \in \mathbb{R}^{\bar{p}+1}$, onde $\bar{\tau}$ é um vetor não nulo.

Usando (4.19), temos $\bar{\tau}^{t} \bar{S}=0$, onde o espaço solução tem dimensão 1. Portanto, temos que $\bar{\tau}$ é proporcional a $\bar{\sigma}$ e, então, tomamos $\bar{\tau}=\bar{\sigma}$. Para determinarmos o sub-vetor $\tau^{t}=\left[\tau_{1}, \ldots, \tau_{p+1}\right]$, precisamos impor a seguinte condição adicional: 


$$
\tau^{t} \mathbf{s}=\sum_{k=1}^{p+1} \tau_{k} s_{k}=-1, \operatorname{com} s^{t}=\left[s_{1}, \ldots, s_{p+1}\right]^{1}
$$

onde $s_{k}=\operatorname{sinal} \sigma_{k}$, $\circ$ qual, considerada a igualdade

$$
\tau^{t} Q=-\bar{\sigma}^{t} \bar{Q},
$$

define um único $\tau$, dado por:

$$
\tau^{t}\left[\begin{array}{ll}
Q & \mathbf{s}
\end{array}\right]=\left[\begin{array}{ll}
\tau^{t} Q & \tau^{t} \mathbf{s}
\end{array}\right]=\left[\begin{array}{cc}
-\bar{\sigma}^{t} \bar{Q} & -1
\end{array}\right]=-\bar{\sigma}^{t}\left[\begin{array}{ll}
\bar{Q} & \bar{s}
\end{array}\right]
$$

Em (4.22), temos:

$$
\bar{\sigma}^{t} \bar{s}=|\bar{\sigma}|=\sum_{i}\left|\sigma_{1}\right|=1 ;
$$

$$
\begin{aligned}
& \text { além disso }[Q \quad s] \text { é não singular e } \bar{s}^{t}=\left[\bar{s}_{1}, \ldots, \bar{s}_{\bar{p}+1}\right] \text {, com } \\
& \bar{s}_{k}=\operatorname{sinal} \bar{\sigma}_{k} .
\end{aligned}
$$

Resumidamente, temos:

1

Definiremos, na demonstração do Teorema da Troca, um conjunto com cardinalldade $q+1$, da seguinte maneira:

$$
\hat{I}=\left\{1_{k}, \frac{-\sigma_{k}}{\tau_{k}}=\lambda\right\}
$$

Essa condição nos garante que este conjunto $\hat{I}$ e não vazio pols:

$$
\begin{aligned}
& \hat{I} \text { seria vazio se: } \tau_{k}=0 \text { para todo } 1<k<p+1 \\
& \sigma_{k} \tau_{k}>0 \text {, para todo } 1 \leq k \leq p+1,
\end{aligned}
$$

mas pela condição (4.20) isto não acontece. 


$$
\left[\begin{array}{cc}
\sigma & \underset{p+1}{0-} \\
& \bar{\sigma}
\end{array}\right] \mathbf{P}=0
$$

considerada a condição $(4.20)$.

Lembrando que $\bar{b}=d-v \hat{y}$ e $d_{i_{k}}=b_{j_{k}}$ com, $j_{k}=t+\bar{i}_{k}$, reescrevemos (4.17) da seguinte forma:

$$
\bar{\alpha}=\sum_{k=1}^{\bar{p}+1} \bar{\sigma}_{k} b_{j k}-\bar{\sigma}^{t} \bar{Q} \hat{y},
$$

onde $\bar{Q} e ́$ a submatriz de $V$ associada a $\bar{I}$.

Do resultado apresentado em (4.21), segue que:

$$
\bar{\alpha}=\sum_{k=1}^{\bar{p}+1} \bar{\sigma}_{k} b_{j_{k}}+\tau^{t} Q \hat{y} .
$$

Por (4.7), a k-ésima componente de $\hat{Q y}$ é $b_{i_{k}}-s_{k} \alpha$; assim usando (4.20), temos:

$$
\bar{\alpha}=\sum_{k=1}^{\bar{p}+1} \bar{\sigma}_{k} b_{j_{k}}+\sum_{k=1}^{p+1} \tau_{k}\left(b_{i_{k}}-s_{k} \alpha\right),
$$

ou seja,

$$
\bar{\alpha}=\sum_{k=1}^{\bar{p}+1} \bar{\sigma}_{k} b_{j_{k}}+\sum_{k=1}^{p+1} \tau_{k} b_{i_{k}}-\sum_{k=1}^{p+1} \tau_{k} s_{k} \alpha,
$$

ou, ainda, 


$$
\bar{\alpha}=\alpha+\sum_{k=1}^{\bar{p}+1} \bar{\sigma}_{k} b_{j k}+\sum_{k=1}^{p+1} \tau_{k} b_{i_{k}}
$$

Uma troca entre $I$ e $\bar{I}$, pode ser realizada de acordo com a regra a ser descrita no Teorema da Troca.

Nesse teorema, denotamos por $J=\left\{j_{1}, \ldots, j_{-p+1}\right\} \circ H$-Conjunto $\bar{I}$, no qual os elementos são referentes aos indices da matriz $A$, isto é, $j_{k}=t+\bar{i}_{k}$, para $1 \leq k \leq \bar{p}+1$.

TEOREMA 3 - TEOREMA DA TROCA: Para $\bar{\alpha}>\alpha$, um subconjunto não nulo $\hat{I}$ de I pode ser trocado com J para formar um novo H-Conjunto Minimal

$$
I^{*}=(I \backslash \hat{I}) \cup J
$$

para a matriz A com um desvio crescente $\alpha^{*}>\alpha$.

Prova:

Consideremos uma combinação I inear dos dois vetores do $\operatorname{Ker}\left(P^{t}\right)$ dados em (4.23) para determinarmos um outro H-Conjunto Minimal para A:

$$
1\left[\sigma^{t}, 0^{t}\right]^{t}+\theta\left[\tau^{t}, \bar{\sigma}^{t}\right]^{t}=\left[\sigma^{t}+\theta \tau^{t}, \theta \bar{\sigma}^{t}\right]^{t} \in \operatorname{Ker}\left(P^{t}\right)
$$

então,

$$
\left[\sigma^{t}+\theta \tau^{t}, \theta \bar{\sigma}^{-t}\right] P=0
$$

onde o parâmetro $\theta$ é escolhido de modo a cancelar algumas componentes de $\sigma^{t}+\theta \tau^{t}$. 
Tomemos $\theta=\lambda$, onde $\lambda$ é o menor número positivo em

$$
\left\{\frac{-\sigma_{k}}{\tau_{k}} / 1 \leq k \leq p+1\right\} .
$$

Definimos o seguinte conjunto:

$$
\hat{I}=\left\{i_{\cdot k} / \frac{\sigma_{k}}{\tau_{k}}=\lambda\right\} ;
$$

assumimos a cardinalidade de $\hat{I}=q+1$ e $\hat{I}$ não vazio, como foi visto em (4.20).

Mostraremos, agora, que $I^{*}=\{I \backslash \hat{I}\} U J$ é um H-Conjunto Minimal para $A$ através da aplicação da Propriedade 1.

-Primeiramente mostremos que o vetor $\sigma^{*}$ tem componentes não nulas.

De fato, fazendo $\theta=\lambda$ em (4.25) e descartando as $q+1$ componentes nulas de $\sigma^{t}+\theta \tau^{t}$, juntamente com as $q+1$ linhas associadas de $P$, obtemos $\sigma^{*} \mathbf{S}^{*}=0, \operatorname{com} \sigma^{*} \in \mathbb{R}^{*}+1, \quad S \in \mathbb{R}^{\left(p^{*}+1\right) \times n}, p^{*}=p+\bar{p}-q \quad e$ cardinalidade de $I^{*}=p^{*}+1$. Portanto, o vetor $\sigma^{*}$ tem componentes não nulas.

- Mostremos, agora, que posto $\left(\mathbf{S}^{*}\right)=\mathrm{p}^{*}$.

Consideremos uma combinação linear das primeiras $\mathrm{p}^{*}$ linhas de $\mathrm{S}^{*} \mathrm{~K}$, que tem o mesmo posto de $\mathbf{S}^{*}$.

Por (4.19) podemos escrever: 


$$
\left[\begin{array}{lll}
u^{t} & v^{t} & o^{t}
\end{array}\right]\left[\begin{array}{cc}
Q^{1} & 0_{p-q, \bar{n}} \\
& \bar{Q}
\end{array}\right]=0,
$$

onde $Q^{1}$ é a submatriz de $Q$ correspondente a $I \backslash \hat{I}, u \in \mathbb{R}^{p-q}$ e $v \in \mathbb{R}^{\bar{p}}$.

Esta relação implica que

$$
\left[\begin{array}{ll}
\mathbf{v}^{\mathrm{t}} & 0^{\mathrm{t}}
\end{array}\right] \overline{\mathbf{s}}=0,
$$

e pela propriedade 4, temos $\mathbf{v}=0$

Temos, então, que $u^{t} Q^{1}=0$ e, pela mesma propriedade, segue $u=0$. Portanto, posto $\left(\mathbf{S}^{*}\right)=\mathrm{p}^{*}$.

Provaremos, agora, que o desvio $\alpha^{*}$ em I é maior que $\alpha$.

Juntamente com (4.25), consideremos a seguinte função:

$$
\alpha(\theta)=\frac{\left|\sum_{k=1}^{p+1}\left[\sigma_{k}+\theta \tau_{k}\right] b_{i k}+\sum_{k=1}^{p+1} \theta \bar{\sigma}_{k} b_{j k}\right|}{\sum_{k=1}^{p+1}\left|\sigma_{k}+\theta \tau_{k}\right|+\sum_{k=1}^{p+1}\left|\theta \bar{\sigma}_{k}\right|}
$$

onde devido a (4.8) e (4.24), o numerador é:

$$
\left|\sum_{k=1}^{p+1} \sigma_{k} b_{i_{k}}+\sum_{k=1}^{p+1} \theta \tau_{k} b_{i_{k}}+\sum_{k=1}^{\bar{p}+1} \theta \bar{\sigma}_{k} b_{j_{k}}\right|=
$$




$$
\begin{aligned}
& =\left|\sum_{k=1}^{p+1} \sigma_{k} b_{i k}+\theta\left[\sum_{k=1}^{p+1} \tau_{k} b_{i k}+\sum_{k=1}^{p+1} \bar{\sigma}_{k} b_{j k}\right]\right|= \\
& =|\alpha+\theta(\bar{\alpha}-\alpha)| .
\end{aligned}
$$

Para $0 \leq \theta \leq \lambda$, o sinal de $\sigma_{k}+\theta \tau_{k}$ é igual ao $\operatorname{sinal}\left(\sigma_{k}\right)=s_{k}$, de modo que, por (4.20) obtemos:

$$
\begin{aligned}
\sum_{k=1}^{p+1}\left|\sigma_{k}+\theta \tau_{k}\right| & =\sum_{k=1}^{p+1} s_{k}\left(\sigma_{k}+\theta \tau_{k}\right)= \\
& =\sum_{k=1}^{p+1} s_{k} \sigma_{k}+\sum_{k=1}^{p+1} s_{k} \theta \tau_{k}= \\
& =\sum_{k=1}^{p+1} s_{k} \sigma_{k}+\theta \sum_{k=1}^{p+1} s_{k} \tau_{k}=1-\theta .(4.28)
\end{aligned}
$$

Por outro lado, temos:

$$
\begin{aligned}
\sum_{k=1}^{\bar{p}+1}\left|\theta \bar{\sigma}_{k}\right| & =\sum_{k=1}^{\bar{p}+1}|\theta|\left|\bar{\sigma}_{k}\right|= \\
& =|\theta| \sum_{k=1}^{\bar{p}+1}\left|\bar{\sigma}_{k}\right|=|\theta|=\theta
\end{aligned}
$$

pois $0 \leq \theta \leq \lambda$.

De (4.28) e (4.29), temos que o denominador de (4.26) se reduz à unidade e, considerando (4.27) o desvio $\alpha^{*}$ em $I^{*}$ é dado por: 


$$
\alpha(\lambda)=\alpha+\lambda(\bar{\alpha}-\alpha)
$$

o que prova o teorema.

\subsection{Determinação do H-Conjunto Minimal.}

Para aplicarmos a divisão do sistema da seção 4.4, necessitamos de um H-Conjunto Minimal para a matriz A, a qual é suposta tendo linhas não nulas. Nesta seção fornecemos um procedimento para a determinação de um $\mathrm{H}$-Conjunto Minimal, o qual depende dos valores relativos de $m$ e $n$ e nosso objetivo é maximizar $\alpha$.

Vejamos o procedimento para dois casos separadamente:

CASO 1: $\mathrm{m}>\mathrm{n}$.

Sabemos que o desvio $\alpha$ em $I=\left\{i_{1}, \ldots, i_{p+1}\right\}$, dado por (4.8), é limitado pelo $\max _{i \in}\left|b_{i}\right|$. Podemos maximizar este limite seguindo os seguintes passos:

i) Selecionamos um conjunto de indices relacionado ao maior valor absoluto das componentes do vetor $b$, com cardinalidade $(n+1)$, devido à Propriedade 2;

ii)Tomamos uma submatriz $B \in \mathbb{R}^{(n+1) \times n}$ de $A$, onde suas linhas são as correspodentes aos indices do conjunto anterior, com posto(B) $=u$, pois a matriz A tem linhas não nulas, por hipótese. Aplicamos a decomposição (4.11) e obtemos: 


$$
\left[\begin{array}{ll}
Y^{-1} & 0_{u, n+1-u} \\
-L & I_{n+1-u}
\end{array}\right] B=\left[\begin{array}{ll}
I_{u} & Z \\
0_{n+1-u, u} & 0_{n+1-u, n-u}
\end{array}\right] \text {. }
$$

onde $Y \in \mathbb{R}^{u \times u}, L \in \mathbb{R}^{(n+1-u) \times u}$ e $Z \in \mathbb{R}^{u x(n-u)}$.

Observamos que cada coluna de $\left[-L \quad I_{1}\right]^{t} \in \mathbb{R}^{(n+1) \times(n+1-u)}$ é um vetor da base do $\operatorname{Ker}\left(B^{t}\right)$, onde um desvio pode ser calculado usando (4.8).

iii)Selecionamos uma coluna que produza o desvio máximo e descartamos todas as colunas de $B^{t}$ que estão associadas com uma coordenada nula deste vetor do $\operatorname{Ker}\left(B^{t}\right)$.

1v)Devido a Propriedade 1, a submatriz resultante B é a matriz $S \in \mathbb{R}^{(p+1) \times n}$ de um $H$-Conjunto Minimal $I=\left\langle i_{1}, \ldots, i_{p+1}\right\}$ com $p>0$, de acordo com Propriedade 3.

CASO 2: $\mathrm{m} \leq \mathrm{n}$.

Nesse caso, temos que $B=A$ e, portanto, posto $(B)=\operatorname{posto}(A)=r$.

Se $r<m$, usamos a mesma técnica do caso 1 para obter I e S;

Se $r=m$ a matriz tem posto linha completo e portanto não existe H-Conjunto para A. O sistema $\mathbf{A} \mathbf{x}=\mathbf{b}$ é resolvido exatamente pelos vetores (4.16) e o correspondente desvio $\alpha$ é nulo.

$\mathrm{Na}$ próxima seção, veremos o algoritmo do método proposto para a 
resolução de sistemas lineares.

\subsection{Algoritmo e Exemplos Numéricos.}

Apresentamos, agora, o algoritmo para resolução de sistemas de equações lineares e alguns exemplos numéricos.

\subsubsection{Algoritmo.}

Nosso algoritmo usa algumas rotinas, as quais são apresentadas a seguir:

\section{Rotina Zero}

Inlcio

Seleclone $t^{(0)}$ equações relaclonadas a vetores llinhas nulas de $A$;

Particlone A e b

$$
A=\left[\begin{array}{l}
0 \\
A^{(1)}
\end{array}\right], \quad b=\left[\begin{array}{l}
d^{(0)} \\
b^{(1)}
\end{array}\right] ; \operatorname{com} A^{(1)} \in \mathbb{R}^{m^{(1)}} \times n^{(1)} \text { e } b^{(1)} \in \mathbb{R}^{(1)} ; .
$$

$m^{(1)} \leftarrow m-t^{(0)} ;$

$n^{(1)} \leftarrow n$;

Calcule $\alpha^{(0)}=\left\|d^{(0)}\right\|$.

Sistema para resolver $A^{(1)} \mathrm{X} \approx \mathrm{b}^{(1)}$.

Fim. 


\section{Rotina Um}

Inlcio

Método da seção 4.5.

Fim.

\section{Rotina Dois}

Inlcio

Método da seção 4.3 .

Sistema posto completo

$$
\begin{aligned}
& T_{(1)}^{(k)} y_{(1)}^{(k)} \approx c_{(1)}^{(k)} \text {, de } t_{(1)}^{(k)} \text { linhas por } p_{(1)}^{(k)} \text { colunas. } \\
& \text { Calcule } \alpha_{(1)}^{(k)}, \hat{y}_{(1)}^{(k)} \text { em } I_{(i)}^{(k)} \text { usando } b_{1,}-u_{i j}^{t} \hat{x}_{j}=s_{j} \alpha .
\end{aligned}
$$

FIm

\section{Rotina Três}

Inlcio

Rotina dois.

$$
\begin{aligned}
& \text { Enquanto }\left\|c_{(1)}^{(k)}-T_{(1)}^{(k)} \hat{y}_{(1)}^{(k)}\right\| \neq \alpha_{1} \text { faça } \\
& \text { Troca do tipo } 1 \text { entre } I(k) \text { e um lndice extremo, } \\
& \text { obtendo um novo H-Conjunto minimal } I_{(i+1)}^{(k)} \text { com } \\
& p_{(1+1)}^{(k)}>0 \text { e } \alpha_{(1+1)}^{(k)}>\alpha_{(1)}^{(k)} \text {. } \\
& \text { Se } p_{(i+1)}^{(k)}<p_{(i)}^{(k)} \text { então } \\
& \text { Atual bze todas as matrizes } \\
& 1 \leftarrow 1+1
\end{aligned}
$$

Fim enquanto.

$$
\begin{aligned}
& I^{(k)} \leftarrow I_{(1+1)}^{(k)} ; U^{(k)} \leftarrow U_{(1+1)}^{(k)} ; T^{(k)} \leftarrow T_{(1+1)}^{(k)} ; e^{(k)} \leftarrow c_{(1+1)}^{(k)} \\
& \alpha^{(k)} \leftarrow \alpha_{(1+1)}^{(k)} ; t^{(k)} \leftarrow t_{(1+1)}^{(k)} ; \cdot m^{(k)} \leftarrow m_{(1+1)}^{(k)}
\end{aligned}
$$

FIm. 


\section{Rotina Quatro}

Inicio

Método da seção 4.3 para construir o sistema:

$$
\begin{aligned}
& A^{(k)} y b^{(k)} \\
& m^{(k)} \leftarrow m^{(k-1)}-t^{(k-1)} \\
& n^{(k)} \leftarrow n^{(k-1)}-p^{(k-1)}
\end{aligned}
$$

FIm.

\section{Rotina Cinco}

Intcio

$$
\begin{aligned}
& \text { Troca tipo } 2 \text { entre } I^{(k-1)} \text { e } I_{(1)}^{(k)} \text { obtendo um novo } \\
& \text { H-ConJunto Minimal para } A^{(k-1)} \\
& \text { Determinamos } \alpha^{(k-1)} ; \\
& k \leftarrow k-1 ;
\end{aligned}
$$

Fim.

Apresentamos, agora, o algoritmo 


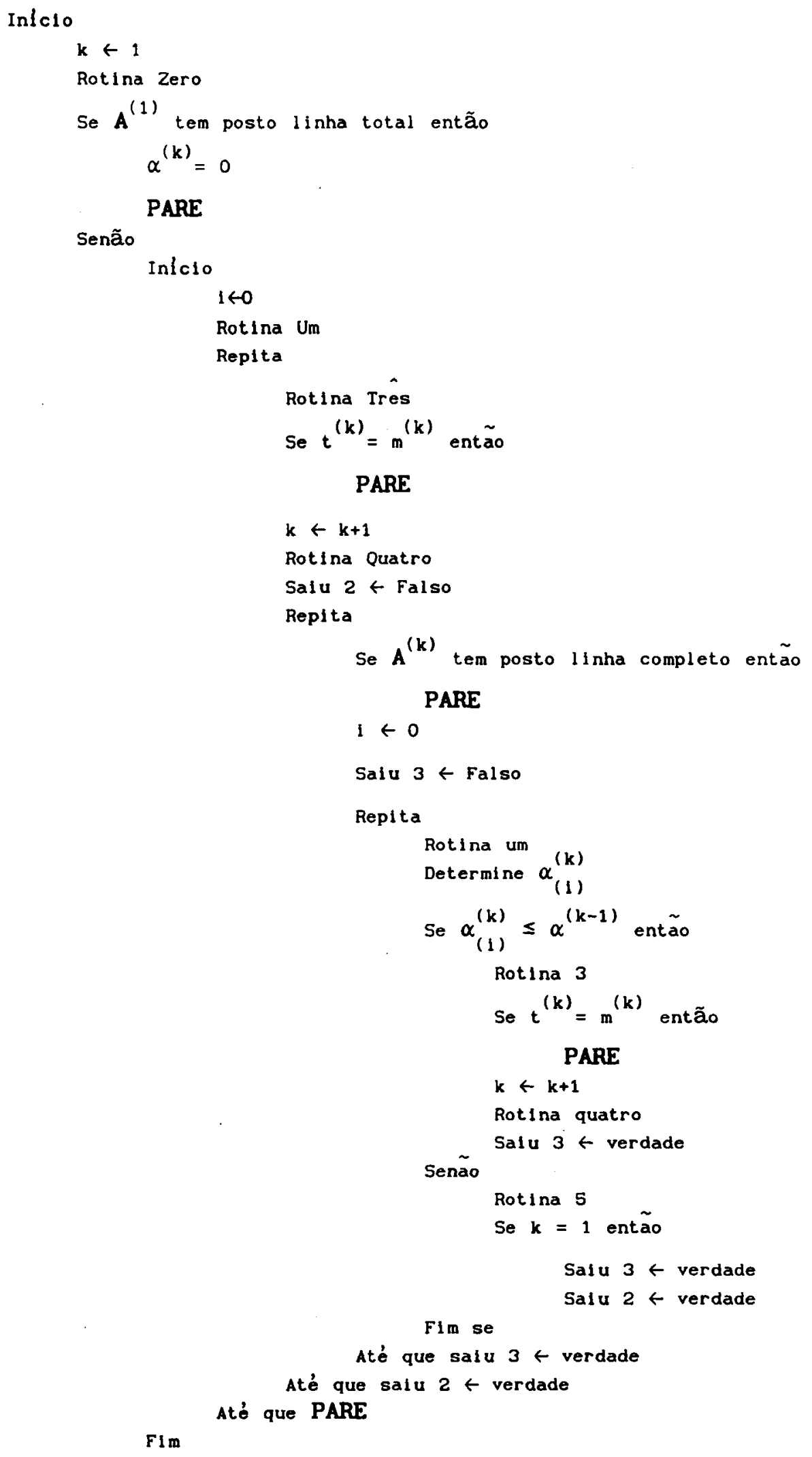

Fim. 
A cada iteração, o algoritmo aumenta o desvio do sistema de índice $\mathrm{k}$ e, além disso, converge para uma sequência de $\mathrm{H}$-Conjuntos Minimais

$$
I^{(1)}, I^{(2)}, \ldots, I^{(g)} \text {, }
$$

e para uma sequência de desvios decrescentes

$$
\alpha^{(1)}, \alpha^{(2)}, \ldots, \alpha^{(9)}
$$

onde cada desvio corresponde a uma solução de Chebyshev do sistema relacionado, dado por:

$$
\alpha^{(k)}=\left\|c^{(k)}-T^{(k) \hat{y}} \hat{y}^{(k)}\right\|, \operatorname{com} 1 \leq k \leq g .
$$

Levando em consideração essas condições, e considerando (4.16) podemos enunciar o seguinte teorema:

TEOREMA 4: A solução de Chebyshev para o sistema $A x \approx b$ é dada por:

$$
\mathbf{x}^{(1)}+H^{(1)}\left[\mathbf{x}^{(2)}+\ldots+\left[\mathbf{x}^{(g-1)}+H^{(g-1)}\left[\mathbf{x}^{(g)}+H^{(g)} v\right)\right] \ldots\right]
$$

onde

$$
x^{(k)}=\left[\begin{array}{c}
\hat{y}^{(k)} \\
0_{n}(k)
\end{array}\right], \quad H^{(k)}=\left[\begin{array}{c}
-U^{(k)} \\
I_{n}(k)
\end{array}\right],
$$

$\operatorname{com} 1 \leq k \leq g$ e $n^{(k)}=n-p^{(1)}-p^{(2)}-\ldots-p^{(k)} ; U^{(k)} \in \mathbb{R}^{p^{(k)}{ }^{(k)}}$ é a matriz relacionada a $I^{(k)}$ e $v$ é algum vetor do $\mathbb{R}^{n-r}$.

Temos, então que a solução é única se e somente se, o sistema for posto coluna completo. Caso contrário, é uma variedade linear, a 
qual é a soma $x+H v$, onde:

$\hat{\mathbf{x}}=\mathbf{x}^{(1)}+\mathbf{H}^{(1)}\left[\mathbf{x}^{(2)}+\ldots+\left[\mathbf{x}^{(g-1)}+H^{(g-1)} \mathbf{x}^{(g)}\right] \ldots\right]=\left[\begin{array}{l}\hat{y} \\ 0 \\ n-r\end{array}\right]$

e

$$
H=H^{(1)} H^{(2)} \ldots H^{(g)}=\left[\begin{array}{c}
-U \\
I_{n-r}
\end{array}\right] \text {, }
$$

$\operatorname{com} \hat{y} \in \mathbb{R}^{r}$ e U $\in \mathbb{R}^{\mathrm{nx}(\mathrm{n}-\mathrm{r})}$, definem completamente a solução.

\subsubsection{Exemplos Numéricos.}

Apresentamos agora algums exemplos que podem ser resolvidos através do algoritmo.

EXEMPLO 1: A matriz A tem posto coluna completo, portanto, a solução é unicamente determinada. Se aproximarmos 1 pelos elementos do espaço $\left\{t, t^{2}\right\}$ no conjunto discreto $\{1,0,-1\}$, produzimos o sistema:

$$
\mathrm{A} \mathbf{x} \approx \mathrm{b}
$$

onde

$$
A=\left[\begin{array}{rr}
1 & 1 \\
0 & 0 \\
-1 & 1
\end{array}\right] \quad \text { e } \quad \mathbf{b}=\left[\begin{array}{l}
1 \\
1 \\
1
\end{array}\right]
$$

Resolução:

Descartamos a linha nula de A, ou seja, a linha 2 , obtendo um novo sistema $A^{(1)} x \approx b^{(1)}$, onde: 


$$
\mathbf{A}^{(1)}=\left(\begin{array}{cc}
1 & 1 \\
-1 & 1
\end{array}\right) \quad \text { e } \mathbf{b}^{(1)}=\left(\begin{array}{l}
1 \\
1
\end{array}\right) \text {. }
$$

Determinamos

$$
I^{(0)}=\{2\} \text { e } \alpha^{(0)}=d^{(0)}=1 \text {. }
$$

Como a matriz $A^{(1)}$ é não singular, o sistema portanto tem solução única, dada por

$$
\hat{\mathbf{x}}^{t}=(1,1),
$$

$\operatorname{com} I^{(1)}=\{1,3\}$ e $\alpha^{(1)}=0$.

Observamos que o conjunto $I^{(1)}$ não é um H-Conjunto para A.

EXEMPLO 2: Se a matriz A tem posto completo e os desvios são tais que $\alpha^{(0)} \leq \alpha^{(1)}=\alpha^{(2)}=\ldots=\alpha^{(g)}=\alpha$, temos a unicidade da solução de Chebyshev. Isto acontece quando aproximamos $t_{1}^{2}+t_{2}^{2}$ por um polinômio de grau dois no seguinte espaço $\left\{1, t_{1}, t_{2}\right\}$. No conjunto $\{(0,0),(-1,0),(1,0),(0,1),(0,-1)\}$, obtemos, o sistema:

$$
A \mathbf{x} \approx b
$$

onde $A=\left(\begin{array}{rrr}1 & 0 & 0 \\ 1 & -1 & 1 \\ 1 & 1 & 1 \\ 1 & 0 & 1 \\ 1 & 0 & -1\end{array}\right)$ e $\quad \mathbf{b}=\left(\begin{array}{l}0 \\ 1 \\ 1 \\ 1 \\ 1\end{array}\right)$ 
Resol ução:

Aplicando o processo da seção 4.5 temos:

$$
B=\left[\begin{array}{rrr}
1 & -1 & 0 \\
1 & 1 & 0 \\
1 & 0 & 1 \\
1 & 0 & -1
\end{array}\right]=\left[\begin{array}{l}
Y \\
L Y
\end{array}\right.
$$

onde $L=\left[\begin{array}{lll}1 & 1 & -1\end{array}\right]$

Escrevendo B como (4.30), temos:

$$
\begin{gathered}
\sigma^{(1)^{\mathrm{t}}}=(-1,-1,+1,+1), \\
\alpha^{(1)}=0, \\
I^{(1)}=\{2,3,4,5\},
\end{gathered}
$$

logo,

$$
\mathbf{S}=\left[\begin{array}{rrr}
1 & -1 & 0 \\
1 & 1 & 0 \\
1 & 0 & 1 \\
1 & 0 & -1
\end{array}\right]=\mathbf{Q}
$$

Apl icando o procedimento do seção 4.3, obtemos:

$$
\mathbf{A}=\left[\begin{array}{rrr}
1 & -1 & 0 \\
1 & 1 & 0 \\
1 & 0 & 1 \\
1 & 0 & -1 \\
\cdots & 0 & 0
\end{array}\right]=\left[\begin{array}{c}
\mathbf{s} \\
\ldots \ldots \ldots \ldots \\
\mathbf{S}
\end{array}\right]
$$


Usando equação $b_{1, j}-u_{i}^{t} \hat{x}^{(1)}=s_{j} \alpha$, vem:

$$
\left[\begin{array}{l}
1 \\
1 \\
1 \\
1
\end{array}\right]-\left[\begin{array}{rrr}
1 & -1 & 0 \\
1 & 1 & 0 \\
1 & 0 & 1 \\
1 & 0 & -1
\end{array}\right]\left[\begin{array}{l}
x_{1} \\
x_{2} \\
x_{3}
\end{array}\right]=\left(\begin{array}{r}
-1 \\
-1 \\
1 \\
1
\end{array}\right] 0
$$

de onde segue que:

$$
x^{(1)^{t}}=(1,0,0) \text { e } r(\hat{x})^{t}=(-1,0,0,0,0) .
$$

Portanto,

$$
\|r(\hat{x})\| \geq \alpha^{(0)}
$$

e $i=1$ entra no conjunto $I^{(1)}$.

Usando (4.22), temos, que

$$
\tau^{t}=\left(0,0,-\frac{1}{2},-\frac{1}{2}\right)
$$

e

$$
\hat{I}=\{4,5\}
$$

Assim, o novo H-Conjunto Minimal é dado por $I^{(1)}=\{1,2,3\}$, ० que acarreta

$$
\mathbf{S}=\left[\begin{array}{rr:r}
1 & 0 & 0 \\
1 & -1 & 0 \\
1 & 1 & 0
\end{array}\right]=\left[\begin{array}{l:l}
\mathbf{Q} & \mathbf{R}
\end{array}\right]
$$

Usando o procedimento do seção 4.5 temos: 


$$
\mathbf{S}=\left(\begin{array}{rr:c}
1 & 0 & 0 \\
1 & -1 & 0 \\
\hdashline 1 & 1 & 0
\end{array}\right]=\left[\begin{array}{c:c}
Y & Y Z \\
\ldots \ldots \ldots & \ldots . \\
L Y & L Y Z
\end{array}\right]
$$

onde $Z=\left[\begin{array}{l}0 \\ 0\end{array}\right]$ e $L=\left[\begin{array}{ll}2 & -1\end{array}\right]$.

Por (4.30), temos:

$$
\sigma^{(1)^{t}}=(-2,1,1) \text { e } \alpha^{(1)}=\frac{1}{2} \text {. }
$$

Utilizando a partição da seção 4.3 , podemos escrever:

$$
\mathbf{A} \mathbf{K}=\left(\begin{array}{rr:r}
1 & 0 & 0 \\
1 & -1 & 1 \\
1 & 1 & 1 \\
\hdashline 1 & 0 & 1 \\
1 & 0 & -1
\end{array}\right)=\left[\begin{array}{c:c}
\mathbf{T} & 0 \\
\ldots \ldots & \ldots . \\
\mathbf{V} & \mathrm{W}-\mathrm{VU}
\end{array}\right)
$$

Resolvendo o sistema $T y^{(1)} \approx c$, onde $c^{t}=(0,1,1)$ e $y^{(1)}=\left(x_{1}, x_{2}\right)$, otemos:

$$
y^{(1)}=\left[\frac{1}{2}, 0\right]
$$

Como $\left\|c-T y^{(1)}\right\|=\alpha^{(1)}=\frac{1}{2}$, tomamos um novo $\mathrm{H}$-Conjunto Minimal $I^{(2)}=\{4,5\}$, onde,

$$
A^{(2)}=\left[\begin{array}{c}
1 \\
-1
\end{array}\right] \text { e } b^{(2)}=\left[\begin{array}{l}
\frac{1}{2} \\
\frac{1}{2}
\end{array}\right] \text {, }
$$

de onde vem que 


$$
\sigma^{(2)^{t}}=(1,1), \alpha^{(2)}=\frac{1}{2} \text { e } x^{(2)^{t}}=x_{3}=0
$$

Portanto, como

$$
\alpha^{(1)}=\alpha^{(2)}=\frac{1}{2}
$$

temos a solução,

$$
\hat{\mathbf{x}}^{t}=\left[\frac{1}{2}, 0,0\right)
$$

com o resíduo:

$$
\mathbf{r}(\hat{x})=\left[-\frac{1}{2}, \frac{1}{2}, \frac{1}{2}, \frac{1}{2}, \frac{1}{2}\right] .
$$

EXEMPLO 3: Para sistemas com posto coluna deficiente, a aproximação é uma variedade linear, conforme (4.32). Resolvemos o sistema:

$$
\mathbf{A} \mathbf{x} \approx \mathbf{b} \text {, }
$$

onde

$$
\mathbf{A}=\left[\begin{array}{cccccc}
1 & 1 & 1 & 1 & 1 & 1 \\
0 & 1 & 1 & 1 & 1 & 1 \\
0 & 0 & 0.2 & 0.2 & 0.2 & 0.2 \\
0.01 & 0 & 0 & 0 & 0 & 0
\end{array}\right] \text { e } \mathbf{b}=\left(\begin{array}{c}
-8 \\
0 \\
-0.2 \\
0
\end{array}\right]
$$

que exemplifica essa situação.

\section{Resolução:}

Aplicamos o procedimento da seção 4.5 para decompor a matriz A, 
produzindo:

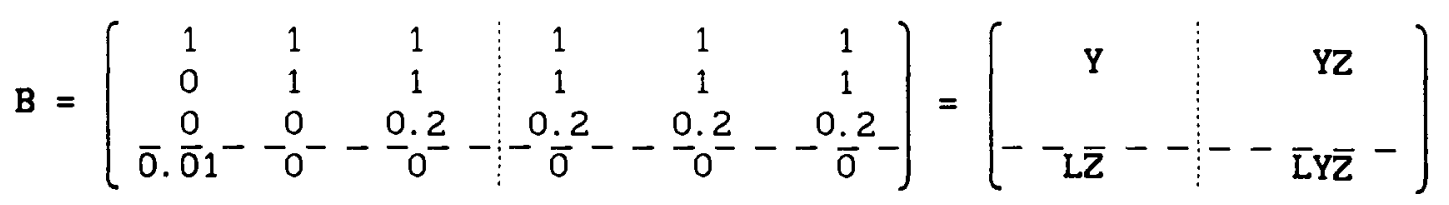

Escrevendo as três últimas colunas de B como combinação linear da base formada pelas duas primeira, obtemos:

$$
Z=\left[\begin{array}{lll}
0 & 0 & 0 \\
0 & 0 & 0 \\
1 & 1 & 1
\end{array}\right]
$$

Do mesmo modo, para a última linha, temos:

$$
L=\left[\begin{array}{lll}
0.01 & -0.01 & 0
\end{array}\right]
$$

Como a terceira componente de $L$ é nula, descartamos a terceira linha de B, obtendo, então, de acordo com (4.13), as matrizes:

$$
I^{(1)}=\{1,2,4\}, \quad S=\left[\begin{array}{cc:cccc}
1 & 1 & 1 & 1 & 1 & 1 \\
0 & 1 & 1 & 1 & 1 & 1 \\
0.01 & 0 & 0 & 0 & 0 & 0
\end{array}\right] \text { e } \sigma^{t}=\left(\begin{array}{lllll}
-0.01 & 0.01 & 1
\end{array}\right),
$$

onde $\mathbf{Q}=\left(\begin{array}{cc}1 & 1 \\ 0 & 1 \\ 0.01 & 0\end{array}\right)$

Escrevendo as últimas colunas de $\mathbf{S}$ em termos da base formada pelas duas primeiras, obtemos: 


$$
\mathbf{U}=\left[\begin{array}{llll}
0 & 0 & 0 & 0 \\
1 & 1 & 1 & 1
\end{array}\right)
$$

e de $\sigma^{t}$, obtemos o vetor sinal $s^{t}=(-1,1,1)$.

Aplicando a decomposição da seção 4.3 à matriz A obtemos,

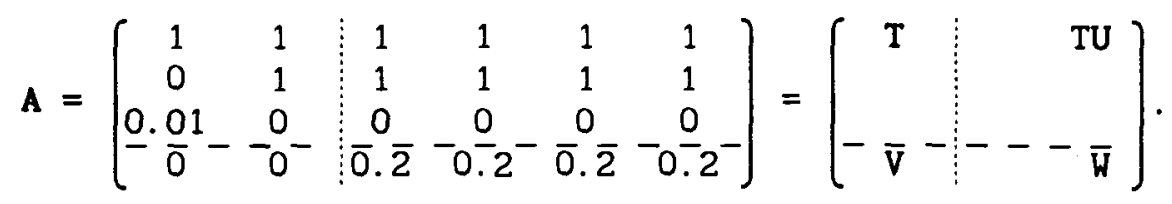

Resolvendo o sistema

$$
\text { (Q s) } \hat{\mathbf{x}}^{(1)}=\mathbf{b} \text {, }
$$

onde $(\mathbf{Q} \mathbf{s})=\left[\begin{array}{ccc}1 & 1 & -1 \\ 0 & 1 & 1 \\ 0.01 & 0 & 1\end{array}\right], \hat{y}^{(1)}=\left[\begin{array}{l}\hat{x}_{1} \\ \hat{x}_{2} \\ \alpha^{(1)}\end{array}\right], \quad \mathbf{b}=\left[\begin{array}{c}-8 \\ 0 \\ 0\end{array}\right]$ e $\hat{x}^{(1)}=$

obtemos

$$
\hat{y}^{(1)^{t}}=\left[-\frac{8}{1.02},-\frac{0.08}{1.02}, \frac{0.08}{1.02}\right] .
$$

Como

$$
\left\|\mathbf{c}-\hat{\mathrm{Tx}}^{(1)}\right\|_{\infty}=\frac{0.08}{1.02}=\alpha^{(1)} \text {, }
$$


segue que $I^{(2)}=\{3\}$. Uma nova aplicação do processo da seção 4.3, resulta em um novo sistema, dado por:

$$
A^{(2)} \hat{x}^{(2)}=b^{(2)},
$$

onde

$$
A^{(2)}=\left[\begin{array}{llll}
0.2 & 0.2 & 0.2 & 0.2
\end{array}\right] \text { e } b^{(2)}=-0.2 .
$$

Como a matriz tem posto linha completo, temos que

$$
\alpha^{(2)}=0 \text {, }
$$

e uma solução para o sistema é dada por

$$
\hat{x}^{(2)^{t}}=\left(x_{3}, x_{4}, x_{5}, x_{6}\right)=(-1,0,0,0)
$$

Logo a solução de Chebyshev é dada por:

$$
\hat{\mathbf{x}}=\hat{\mathbf{x}}^{(1)}+\mathrm{H}^{(1)}\left[\hat{\mathbf{x}}^{(2)}+\mathrm{H}^{(2)} \mathrm{v}\right]
$$

onde $\hat{\mathbf{x}}^{(1)}$ e $\hat{\mathbf{x}}^{(2)}$ já foram determinados e

$$
H^{(1)}=\left[\begin{array}{c}
-U^{(1)} \\
I_{4}
\end{array}\right] \text { e } \quad H^{(2)}=\left(\begin{array}{c}
-U^{(2)} \\
I_{3}
\end{array}\right) \text {. }
$$


Logo,

$$
\hat{\mathbf{x}}=\left[\begin{array}{c}
\frac{-8}{1.02} \\
\frac{0.94}{1.02} \\
-1 \\
0 \\
0 \\
0
\end{array}\right]+\left[\begin{array}{rrr}
0 & 0 & 0 \\
0 & 0 & 0 \\
-1 & -1 & -1 \\
1 & 0 & 0 \\
0 & 1 & 0 \\
0 & 0 & 1
\end{array}\right] v
$$

e

$$
r(\hat{x})^{t}=\left[\frac{0.08}{1.02}, \frac{0.08}{1.02}, 0, \frac{0.08}{1.02}\right] .
$$

Os exemplos aqui apresentados, são pequenos e não foram resolvidos com auxilio do computador, pois, optamos por implementar a especialização do método Simplex, como visto no Capítulo 3 , ao invés de implementar o algoritmo dessa seção. 


\section{CONCLUSÕES}

Vimos, neste trabalho, que o problema de determinar a solução aproximada de um Sistema Linear Inconsistente pode ser resolvido através de um problema de Programação Linear.

Em relação ao estudo de caso, Planicidade de Superficles, verificamos que a especialização do método Simplex produz, sempre, uma solução "melhor" do que a fornecida pelo método dos Minimos Quadrados. Além disso, ressaltamos a simplicidade de aplicação do método Simplex, a estrutura de dados do problema que nos permite economizar memória, devido ao fato de podermos gerar as linhas da matriz das restrições e a economia de tempo computacional obtida, ao selecionarmos colunas linearmente independentes associadas aos maiores valores das medições. Entretanto, o tempo computacional do método dos Mínimos Quadrados é "menor", mas levando em consideração que a solução fornecida pelo método Simplex é melhor, as diferenças entre os métodos e o tempo gasto para obter as medições ser grande, concluímos que a diferença de tempo é totalmente aceitável.

Do ponto de vista da Planicidade de Superficies, é considerado um erro de 3\% para a solução fornecida por qualquer método, devido a "Erros de Medição". Portanto, só condideramos que a solução fornecida pelo algoritmo da seção 3.5 é signiflcativamente melhor do que a dos Mínimos Quadrados, se a diferença entre elas é de pelo menos 3\%. Por isso, na seção 3.6, fizemos algumas varlações nas medições feitas em [11], e observando as tabelas, podemos verificar que a condição dos $3 \%$ foi satisfeita nos exemplos de 2 à 7 . 
Finalmente, no Capítulo 4, apresentamos a revisão do artigo [12], - qual explora decomposições da matriz e partições do sistema, onde fornecemos a prova de vários teoremas propostos, bem como o algoritmo do método estruturado. Ressaltamos que este algoritmo, do ponto de vista computacional, é dispendioso, devido às suas comparações de soluções e trocas. 
BIBLIOGRAFIA

[ 1] DESCLOUX, J. Approximations in $\mathrm{L}^{\mathrm{p}}$ and Chebyshev approximations. J. Siam, 11, p. 1017-1026, 1963.

[ 2] DURIS, C. \& TEMPLE, M.G. A finite step algorithm for determining the strict Chebyshev solution to $A x=b$. Siam J. Numer. Anal., 10, p.690-699, 1973.

[ 3] FRANCELIN, A.P. Aproximação da solução de sistemas lineares inconsistentes na norma de Chebyshev. ICMSC-SÃO CARLOS, 1986, 76. (Dissertação de Mestrado).

[ 4] HUANG, S.T.; FAN, K.C. \& WU, J.H. A new minimum zone method for evaluating flatness errors. Precision Engineering, 15, p.25-32, 1993.

[ 5] NOBLE, B. A method for computing the generalized inverse of a matriz. SIAM J. Numer. Anal., 3, p.582-584, 1966.

[ 6] OSBORNE, M.R. \& WATSON, G.A. On the best linear Chebyshev approximation. Comp. J., 10, p. 172-177, 1967.

[ 7] POWELL, M.J.D. Approximation theory and methods. London, Cambrige University Press, 1981.

[ 8] RICE, J.R. Tchebycheff approximation in compact metric space. Bull. Amer. Math. Soc., 68, p.405-410, 1962. 
[ 9] RICE, J.R. The Approximation of Functions. Mass, Addison-Wesley, 1969.

[10] RIGHETTO, A. Vetores e Geometria Analitica. Passos, Ivan Rossi, 1976.

[11] SATO, D.P.V. \& BICUDO, L.A.M.C. Medição da Planicidade de um desempeno. São Carlos, 1992. 20p. (Relatório-Faculdade de Engenharia-USP).

[12] THIRAN, J.P. \& THIRY, S. Strict Chebyshev Approximation for General Systems of Linear Equations. Numer. Math, 51, p. 701-725, 1987.

[13] WATSON, G. A. Approximation Theory and Numerical Methods. New York, John Wiley \& Sons, 1980. 